\title{
Mycobacterium abscessus HeIR interacts with RNA Polymerase to confer intrinsic rifamycin resistance
}

40 Keywords: Mycobacterium abscessus, HeID, HeIR, intrinsic resistance, rifampicin, rifabutin,

${ }^{1}$ Division of Genetics, Wadsworth Center, New York State Department of Health, Albany, NY 12208, USA

${ }^{2}$ School of Public Health, University at Albany, Albany, NY 12208

${ }^{*}$ Corresponding author: Pallavi.Ghosh@health.ny.gov

\section{Classification: Biological Sciences/Microbiology}




\section{Abstract}

45 Rifampicin (RIF) constitutes the frontline therapy against $M$. tuberculosis as well as most slow-growing 46 non-tuberculous mycobacteria (NTM). However, RIF is completely ineffective against M. abscessus 47 despite the absence of mutations in the rifampicin resistance determining region of Mab_rpoB. This 48 has been attributed to the presence of an ADP-ribosyltransferase (Arr) activity that inactivates RIF.

49 Rifabutin (RBT), a close analogue of RIF, has recently been shown to be effective against $M$.

50 abscessus in vitro and in a mouse model and comprises a promising therapeutic against $M$.

51 abscessus infections. Using RNA sequencing we show that exposure of $M$. abscessus to sublethal

52 doses of RIF and RBT results in 25-fold upregulation of Mab_helR in laboratory and clinical isolates;

53 an isogenic deletion of Mab_helR is hypersensitive to RIF and RBT, and over-expression of

54 Mab_helR confers RIF tolerance in M. tuberculosis implying that helR constitutes a significant

55 determinant of inducible RIF and RBT resistance. We demonstrate a preferential association of

56 MabHelR with RNA polymerase in vivo in bacteria exposed to RIF and showed that purified MabHelR

57 can rescue transcription inhibition in the presence of RIF in in vitro transcription assays. Furthermore,

58 MabHelR can dissociate RNAP from RIF-stalled initiation complexes in vitro, a species we envisage

59 accumulates upon RIF exposure. Lastly, we show that the tip of the PCh-loop of Mab_helR, in

60 particular residues E496 and D497 that are in proximity to RIF, is critical for conferring RIF resistance

61 without being required for RNAP dissociation from stalled complexes. This suggests that HelR may be additionally involved in displacing RIF bound to RNAP and function as an RNAP protection protein. 


\section{Significance Statement}

65

Bacterial RNA polymerase is a target for the potent and broad-spectrum rifamycin group of antibiotics.

67 Mutations within rpoB and inactivation by a diverse group of enzymes constitute the most widespread

68 mechanisms of resistance. Herein we report an unprecedented mechanism of rifamycin resistance in

69 M. abscessus mediated by MabHelR, a putative SF1 like helicase, that involves disassembly of RIF-

70 stalled initiation complexes, likely followed by displacement of the antibiotic, leading to RNAP

71 recycling. The mechanism is reminiscent of the role of $\mathrm{HflX}$ and ribosome protection proteins in

72 resistance to ribosome targeting antibiotics and suggests that removal of stalled macromolecular

73 complexes and their recycling comprises a widespread but underappreciated mechanism of antibiotic

74 resistance. Rifampicin (RIF) is pivotal in the control of $M$. tuberculosis infections but ineffective against

$75 M$. abscessus. Identification of inducible rifamycin resistance determinants in $M$. abscessus is

76 therefore particularly crucial for informing treatment strategies and development of novel therapeutic

77 approaches. 
Introduction

M. abscessus (Mab) is a rapidly growing non-tuberculous mycobacterium (NTM) and a prominent

cause of broncho-pulmonary infections in patients with chronic lung damage, such as bronchiectasis,

prior tuberculosis and cystic fibrosis (CF) (2013; Catherinot et al., 2013; Martiniano and Nick, 2015;

Martiniano et al., 2014; Roux et al., 2009). M. abscessus also causes superficial and deep-tissue infections post- trauma and surgery (Kothavade et al., 2013; Nakanaga et al., 2011). It is considered an "incurable nightmare" due to its extremely low sensitivity to available antibiotics. (Ballarino et al., 2009;

Brown et al., 1992a; Brown et al., 1992b; Brown-Elliott et al., 2012; Brown-Elliott and Wallace, 2002;

Swenson et al., 1985; Wallace et al., 1991). Notably, M. abscessus displays high levels of intrinsic resistance to the anti-tuberculosis drugs, isoniazid and rifampicin (RIF) (Brown-Elliott et al., 2012; Park et al., 2008). The current treatment regimen of clarithromycin and amikacin in conjunction with cefoxitin, imipenem and tigecycline, for a period of several months (Floto et al., 2016; Griffith et al., 2007) is rendered ineffective by the rapid induction of resistance genes upon antibiotic exposure. against M. tuberculosis and several NTMs (Bittner and Preheim, 2016; Esteban et al., 2012). It is known to bind in a pocket of the $\beta$-subunit (RpoB) of RNA polymerase (RNAP) within the exit tunnel, thereby hindering the passage of nascent RNA when it is greater than 2-3 nt in length and an inhibition of global RNA synthesis. High levels of clinically acquired RIF resistance across bacterial species map within an 81-bp region of $r p o B$ referred to as the $\underline{\text { RIF }}$ Resistance Determining Region (RRDR). Intrinsic RIF resistance in bacteria is attributed to the presence of refractory RNAPs, alteration of cell permeability and the presence of a diversity of enzymes that modify the drug (Goldstein, 2014; Tupin et al., 2010). RIF inactivation by ADP-ribosyl transferases

(Arrs), RIF glycosyltransferases (Rgt), RIF 101 monooxygenase (Rox), and RIF phosphorylases (Rph) have been well characterized in various 102 actinomycetes (Spanogiannopoulos et al., 2014; Tupin et al., 2010). Additionally, the RNAP binding 103 protein, $\mathrm{RbpA}$, has been implicated to play a role in RIF resistance since a Streptomyces coelicolor $104 \Delta r b p A$ mutant is RIF sensitive and overexpression of RbpA results in increased RIF resistance in $M$. 
106 inhibition by RIF on the rrnDp3 promoter in S. coelicolor and in M. smegmatis, (Dey et al., 2010; Newell

107 et al., 2006) subsequent studies by Hu et al.and Hurst-Hess et al. demonstrated that the apparent effect

108 of RbpA on transcription inhibition by RIF was a reflection of significant increase in transcript yield

109 without affecting the $I_{50}$ of RIF(Hu et al., 2012; Hurst-Hess et al., 2019). The effect of RbpA on RIF

110 resistance is therefore indirect and does not involve a hinderance of RIF binding to mycobacterial

111 RNAP (Hu et al., 2012; Hurst-Hess et al., 2019).

112 M. abscessus displays a high level of intrinsic RIF resistance despite lacking mutations in the RRDR

113 of $r p o B$ thereby rendering it unavailable for therapy against M.abscessus infections (Rominski et al.,

114 2017). This has been attributed to the presence of an ADP-ribosyltransferase (Mab_arr) that ribosylates

115 RIF thereby preventing binding to RpoB (Rominski et al., 2017). ADP-ribosyltransferases, while

116 encoded by other fast-growing NTMs such as $M$. smegmatis and $M$. fortuitum, are absent in $M$.

117 tuberculosis, and is the only known determinant of intrinsic RIF resistance in mycobacteria. Rifabutin

118 (RBT), a spiro-piperidyl derivative of RIF typically exhibits lower MIC values for all mycobacteria and

119 has been shown to be effective against $M$. abscessus in vitro and in a mouse model (Aziz et al., 2017;

120 Dick et al., 2020; Ganapathy et al., 2019); RBT therefore constitutes a promising therapeutic option for

$121 M$. abscessus infections. The mechanism underlying the differential potency remains to be determined

122 but is likely a consequence of the higher lipophilicity of the drug (Blaschke and Skinner, 1996).

123 A transcriptomic analysis of $M$. abscessus exposed to sublethal doses of RIF revealed a $>25$-fold

124 induction of MAB_3189c, a putative HelD-like helicase. HelD proteins belong to the SF1 superfamily of

125 RNA and DNA helicases and are widespread in gram positive bacteria (Newing et al., 2020). Previously

126 we showed that exposure of M. smegmatis to sublethal dose of RIF results in a similar upregulation of

127 MSMEG_2174 (Ms_helD), the homologue of MAB_3189c (Hurst-Hess et al., 2019). Due to the

128 involvement of $M s \_h e l D$ and $M A B \_3189 \mathrm{c}$ in resistance to rifamycins, we rename these Ms_helR and

129 Mab_helR respectively. In the present study we demonstrate that a targeted deletion of Mab_helR

130 results in hypersensitivity to both RIF and RBT and investigate the mechanism of resistance. We show

131 that MabHeIR association with RNA polymerase in vitro rescues RIF mediated transcription inhibition

132 and displaces RIF-stalled RNAP from bound promoter DNA. However, PCh-loop mutants of MabHelR 
bioRxiv preprint doi: https://doi.org/10.1101/2021.05.10.443476; this version posted May 10, 2021. The copyright holder for this preprint (which was not certified by peer review) is the author/funder. All rights reserved. No reuse allowed without permission.

133 that are proficient for dissociating stalled RNAP complexes from DNA are incapable of complementing

134 RIF resistance of $\Delta \mathrm{Mab}$ helR suggesting a potentially new mechanism of RIF resistance in which the

135 PCh-loop of MabHelR displaces RIF bound to RNAP. 
RESUTS:

Mab_heIR expression is induced by sublethal concentrations of RIF and RBT

In a preliminary study we used RNA sequencing (RNAseq) to determine the genome-wide

139 changes in gene expression in Mab ATCC 19977 upon exposure to sublethal doses of RIF (16ug/ml for

14030 mins). We observed that six genes were upregulated $>4$-fold $\left(p_{\text {adj }}<0.01\right)$ (Figure 1a). Of these

141 Mab_arr and MAB_3189c showed the greatest changes in expression (>25-fold) which were confirmed

142 using real time PCR (Figure 1b). MAB_3189c is a homologue of HelD proteins that belong to the SF1

143 family of RNA and DNA helicases, and is referred here as helR. Further, we showed that Mab_helR

144 and Mab_arr were similarly upregulated upon exposure to sublethal doses of RBT, a RIF analogue with

145 in vitro activity against $M$. abscessus (Figure 1b). In order to determine the clinical relevance of helR in

146 rifamycin resistance, we followed the RIF/RBT resistance and expression of helR in 8 sequenced clinical

147 isolates of $M$. abscessus obtained from the from the Wadsworth Center Reference Laboratory. The

148 eight isolates included all three subspecies of $M$. abscessus- M. abscessus abscessus, M. abscessus

149 masiliense and M. abscessus bollettii and share 96.6-99.8\% average nucleotide identity (ANI) (Figure

150 1c); all strains displayed rifamycin tolerance similar to that of Mab ATCC (Table S1). As seen in

151 Figure1d, exposure to sublethal concentrations of RIF resulted in upregulation of both Mab_arr and

152 Mab_helR in all eight clinical isolates of $M$. abscessus. The upregulation of Mab_arr upon RIF exposure

153 is consistent with previous studies which show that Arr is a major determinant of RIF resistance in $M$.

154 abscessus (Rominski et al., 2017). A similar upregulation of Mab_helR, in laboratory and clinical strains,

155 is therefore suggestive of a significant role of MabHelR in rifamycin resistance.

157 Deletion of Mab_helR results in hypersensitivity to RIF and RBT

An isogenic deletion of Mab_helR in MabATCC was created using phage recombineering

159 (Hurst-Hess et al., 2017). As seen in Figure 2a, a $\Delta M a b \_h e l R$ strain was hypersensitive to RIF and

160 RBT. Expression of Mab_helR driven by a native promoter from a chromosomally integrated copy in

161 the $\Delta M a b \_h e l R$ strain restored sensitivity of the mutant to wild-type levels (Figure 2a, Table 1). The

162 growth of $\Delta M a b \_h e l R$ in media lacking RIF remained unchanged (Figure S1a). Moreover, a double 
163 mutant of $\Delta M a b \_h e l R$ / $\triangle M a b \_a r r$ was more sensitive than each single mutant alone suggesting that

164 Mab_helR and Mab_arr comprise independent and additive resistance mechanisms (Figure 2b, Table

165 1). Additionally, the expression of Mab_arr remained unchanged in a $\Delta M a b \_h e l R$ background, 166 confirming that the observed hypersensitivity of $\Delta M a b \_h e l R$ to rifamycins is not a consequence of a 167 decrease in expression of Mab_arr in the $\Delta M a b \_h e l R$ strain (Figure 2c).

168 M. tuberculosis H37Rv is highly susceptible to RIF and lacks homologues of Mab_arr and 169 Mab_helR. We expressed Mab_helR in the RIF sensitive M. tuberculosis $m c^{2} 7000$, a severely 170 attenuated derivative of M. tuberculosis H37Rv (Ojha et al., 2008). As seen in Figure 2d, heterologous 171 expression of Mab_helR resulted in increased RIF tolerance of $M$. tuberculosis $m c^{2} 7000$. Furthermore, $172 M$. smegmatis $\Delta M s \_h e l R$ is also RIF sensitive and expression of Mab_helR in a $\Delta M s \_h e l R$ strain 173 restored its antibiotic tolerance to wild-type levels thereby suggesting a conserved function of Mab_helR 174 and Ms_helR (Figure S1b)(Hurst-Hess et al., 2019). These results together establish the role of HelR 175 as an additional mechanism of RIF resistance in mycobacteria and demonstrate that Mab_helR and 176 Mab_arr constitute inducible mechanisms of basal RBT tolerance in M. abscessus.

177 An analysis of upstream sequences of Mab_helR revealed the presence of the RIF associated 178 element (RAE), a highly conserved 19-bp inverted repeat sequence, previously identified upstream of 179 genes encoding RIF-inactivating enzymes (glycosylation, ADP ribosylation and monooxygenation) and 180 helicases from divergent actinomycetes (Spanogiannopoulos et al., 2014) (Figure 2e). The conserved 181 RAEs were found upstream of only Mab_arr and Mab_helR within the $M$. abscessus genome 182 underscoring the importance of helR in rifamycin resistance and suggesting a co-regulation of Mab_arr 183 and Mab_helR by a conserved mechanism. Deletion of one half of the conserved RAE (Figure 2e, 184 shaded in grey) results in the inability to restore RIF sensitivity of the $\Delta$ helR strain (Figure 2f) confirming 185 a role for RAE in the induction of RIF resistance. 
MabHeIR interacts with RNA polymerase in vivo and rescues RIF inhibition of RNAP in in vitro

Previous studies on a putative RAE-associated helicase from Streptomyces WAC4747

demonstrated its inability to inactivate RIF and suggested a mechanism of action other than RIF modification (Spanogiannopoulos et al., 2014). HelD was first detected as a major copurifying protein in preparations of RNA polymerase from $B$. subtilis, and has since been established as a direct binding partner of B. subtilis RNA polymerase involved in RNAP cycling (Newing et al., 2020; Pei et al., 2020; Wiedermannova et al., 2014). Recently, the cryoEM structure of M. smegmatis HelR in complex with RNA polymerase also suggested a role of MsHelR in dissociating stalled elongating RNAP complexes from the associated nucleic acids (Kouba et al., 2020). In order to determine if MabHeIR similarly associates with RNAP, we constructed a MabATCC strain containing a 10X his-tag at the native rpoA locus. MabRNAP his was purified from cells exposed to sublethal quantities of RIF as well as from untreated cells. A band corresponding in size to purified MabHelR was found to be $\sim 5$-fold enriched in RNAP preparations from RIF-exposed cells and was identified as MabHelR using LC-MS mass spectrometry (Figure 3a). Negligible amounts of MabHelR were detectable in RNAP purified from exponentially grown cells in the absence of antibiotic (Figure 3a).

Next, MabHeIR was purified from a MabATCC strain containing a 3X FLAG-tag at the native helR locus exposed to sublethal quantities of RIF, as well as from RIF untreated cells. Western blot analysis with anti-FLAG antibody showed the presence of significant quantities of cellular HelR $\mathrm{FLAG}_{\text {in }}$ antibiotic- untreated M. abscessus which increased in RIF treated cells (Figure 3b). However, interaction of MabHeIR with MabRNAP was detectable using anti-RNAP- $\alpha$ antibody predominantly in RIF-treated cells (Figure 3b). While this does not rule out interaction of HelR with RNAP in exponentially growing bacteria, it demonstrates an enhanced association with RNAP in the presence of RIF.

To validate that purified MabHelR interacts directly with RNAP, we performed in vitro protein-

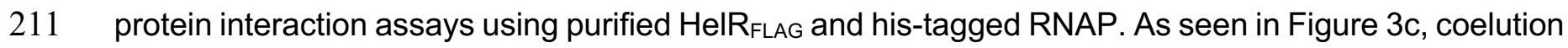

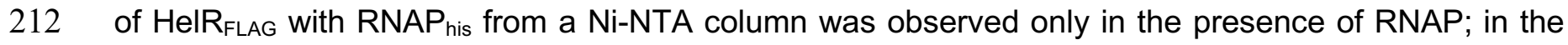




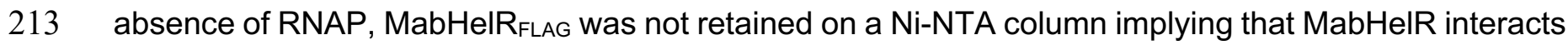

214 directly with RNAP.

215 Finally, since a deletion of HelR results in hypersensitivity to rifamycins, we wished to study the

216 effect of MabHeIR on RIF mediated transcription inhibition. Multiple round in vitro transcription was

217 performed using $M$. tuberculosis RNAP that naturally lacks HelR, and the well- characterized $\sin P 3$

218 promoter (Jacques et al., 2006). A 77nt run-off transcript was observed that diminished in the presence

219 of increasing concentrations of RIF. Addition of a 9-fold or 15-fold molar excess of MabHelR resulted in

220 increased transcript formation at each of the RIF concentrations tested as compared to control reactions

221 lacking HelR suggesting that MabHeIR could rescue transcription inhibition of RNAP by RIF (Figure 3d).

223 The ATPase activity of MabHeIR is required for RIF resistance

224 HelD-like proteins display a four-domain structure containing an N-terminal domain, a 225 superfamily 1 (SF1) $1 \mathrm{~A}$ domain that is split into a $1 \mathrm{~A}-1$ and a $1 \mathrm{~A}-2$ at the primary amino-acid sequence 226 level by a HelD specific domain, and a C-terminal 2A domain. Similar to M. smegmatis HelR, the four 227 conserved structural domains could be discerned in MabHelR (Figure 4a, Figure S2). Domains 1A and 228 2A together form a conserved Rossman fold with seven conserved motifs, including Walker A and 229 Walker B that are required for NTP binding and hydrolysis (Caruthers and McKay, 2002). Purified 230 MabHelR displayed significant ATPase activity that is independent of RNAP (Figure 4b) and was 231 consistent with the behavior of $B$. subtillus HelD (Wiedermannova et al., 2014). In order to determine if 232 the ATPase activity of MabHeIR is required for its ability to confer RIF resistance, we generated two 233 variants of MabHeIR that contained mutations in either the Walker B motifs (MabHelR-mWalkerB) or 234 both Walker $A$ and $B$ motifs (MabHelR-mWalkerAB). Both mutant proteins were defective in ATP 235 hydrolysis in vitro (Figure 4b). Further, MabHelR-mWalkerB and MabHelR-mWalkerAB were unable to 236 restore rifamycin sensitivity of $\triangle M a b \_h e l R$ in vivo despite retaining the ability to bind RNAP (Figure 4c,

237 Figure S3a) implying that ATP hydrolysis is necessary for HelR mediated RIF resistance. Control 238 experiments showed that upon RIF exposure MabHelR-mWalkerB and MabHeIR-mWalkerAB were 239 expressed from the integrated chromosomal copy at levels similar to that of WT HelR (Figure S3b). 
241 The PCh loop of MabHeIR is required for RIF resistance but not for dissociation of RIF-stalled RNAP complexes from DNA

The cryoEM structure of MsHelR revealed a crescent shaped structure; one end of the crescent

244 formed by the $\mathrm{N}$-terminal domain inserts into the secondary channel, while the other end formed by the

245 clamp opening (CO) domain and the primary channel (PCh) loop, inserts into the primary channel

246 (Kouba et al., 2020). Upon binding RNAP, the PCh loop folds into a helix $\alpha 16$ and a helix $\alpha 17$ that pack

247 against the $\beta$ ' bridge helix $(\mathrm{BH})$ and is inserted into the active site (AS) cavity. A model of MabHelR was

248 generated using Phyre v2.0 and superimposed on the structure of MsHelR-RNAP complex (PDB ID

2496 YYS). Superimposition of the crystal structure of M. smegmatis RNAP initiation complex bound to RIF

250 (PDB ID 6CCV) was used to locate RIF on the RNAP-HelR complex (Figure 5a). The tip of the PCh-

251 loop consists of four acidic residues, MabHeIR/494-DDED-497. Of these, residues MabHelR/Asp494

252 and Asp495 are in proximity to MgA and AS conserved motif $\beta$ ' - NADFDGD, while Glu496 and Asp497

253 are in close proximity (within $3.5 \AA$ ) to RIF. Due to the proximity of the PCh-loop to RIF, we sought to

254 investigate the role of the PCh- loop in the ability of MabHelR to confer RIF resistance. For this purpose

255 we generated three mutants of MabHelR; $\triangle \mathrm{PCh} 1$ : deletion in aa 467-515, $\triangle \mathrm{PCh2}$ : deletion in aa 486-

256505 , and mPCh: E496A/D496A. As seen in Figure 5b, deletion of either the entire PCh loop $\alpha 16$ and

$257 \alpha 17(\triangle \mathrm{PCh} 1)$ or the intervening region between the helices $(\triangle \mathrm{PCh} 2)$ results in an inability to restore

258 rifamycin sensitivity of $\triangle M a b \_h e l R$ in vivo, despite retaining the ability to bind RNAP in vitro (Figure

259 S3a). Importantly, alanine substitution of Glu496 and Asp497 is sufficient to result in a marked defect

260 in the ability of the MabHeIR-mPCh to confer RIF resistance (Figure 5b). Control experiments showed

261 that RIF inducible expression of MabHelR $\triangle \mathrm{PCh} 1, \triangle \mathrm{PCh} 2$ and $\mathrm{mPCh}$ in the complementing strains were

262 similar to that of WT MabHeIR (Figure S3b).

Previous studies have shown that the BsuHeID and MsHelR can release stalled RNAP

264 elongation complexes from bound DNA. Binding of RIF to the $\beta$ subunit of RNAP within the DNA/RNA

265 channel sterically blocks the passage of the RNA transcript at the $5^{\prime}$ end when the transcript becomes 
266 either 2 or $3 \mathrm{nt}$ in length, without affecting substrate binding, catalytic activity or translocation (Campbell 267 et al., 2001). RNAP inhibited in the presence of RIF conceivably constitute stalled initiation complexes 268 that could be targets of HelR. In order to determine if MabHeIR can dissociate RIF stalled RNAP 269 complexes from bound promoter DNA, we assembled initiation complexes on a 5'-biotinylated $\sin P 3$ 270 template stalled in the presence of RIF and the initiating nucleotide, ATP. The DNA-RNAP complex was 271 tethered on streptavidin agarose beads and the dissociation of stalled RNAP from promoter DNA was 272 studied as a function of MabHelR addition using western blot analysis with anti-his antibodies that 273 recognize RpoA $A_{\text {his }}$ (Figure 5c). MabHelR was able to dissociate stalled RIF-RNAP complexes from DNA 274 independent of the presence of ATP which is consistent with previous experiments with stalled $M$. 275 smegmatis elongation complexes (Figure 5d) (Kouba et al., 2020). Additionally, the mPCh mutant was 276 also proficient in disassembly of stalled RIF-RNAP complexes despite its inability to complement the 277 RIF sensitivity of the $\Delta M a b \_$helR mutant, suggesting that HelR mediated RIF resistance requires a 278 step in addition to displacement of stalled initiation complexes, likely the active displacement of RIF 279 mediated by interactions with the PCh-loop. 


\section{Discussion}

Enzymatic inactivation of RIF by the rifampicin ADP-ribosyl transferase (arr) is known to be the

282 primary determinant that contributes to the high levels of innate RIF resistance of $M$. abscessus. In

283 the present study we identify a new and significant determinant of inducible RIF/RBT resistance in $M$.

284 abscessus, MabHelR, that functions via an arr independent pathway. We demonstrate that unlike

285 BsuHeID, which is seen to associate in stoichiometric amounts with RNAP isolated from exponentially

286 growing bacteria (Wiedermannova et al., 2014), MabHeIR preferentially associates with RNAP

287 isolated from RIF treated samples (Figure $3 a-b)$. The increased association of MabHeIR with RNAP

288 from RIF treated bacteria could be a mere reflection of increased transcription of Mab_helR upon RIF

289 exposure. However, we note that the amount of cytosolic MabHelR protein does not show an increase

290 proportionate to Mab_heIR transcription upon drug addition (Figure 3b). The inability to detect an

291 interaction of HelR with RNAP in exponentially growing bacteria despite the presence of significant

292 quantities of cytosolic HelR suggests that RIF bound RNAP constitutes a prime target of MabHelR.

293 The cryo-EM structure of MsHelR bound to RNAP reveals that the N-terminal domain is

294 inserted into the secondary channel of RNAP in a way that interferes with the nucleotide addition

295 cycle, and the primary channel is occupied by the PCh-loop and the CO domain (Kouba et al., 2020).

296 The PCh-loop penetrates into the AS and leads to a repulsion of the dsDNA and RNA/DNA hybrid at

297 the AS while the CO domain binds the $\beta$ '-clamp thereby splaying open the RNAP primary channel.

298 Together, these interactions lead to spillage of nucleic acid contents from a transcription complex. The

299 interaction of HelR with RNAP is conceivably inhibitory for transcription and is thought to be required

300 for dissociation of RNAP complexes that are stalled either during elongation or termination. We

301 hypothesized this HelR-RNAP interaction can be extended to the RNAP holoenzyme stalled during

302 initiation in the presence of transcription inhibitors like RIF (Figure 6, complex A), a scenario

303 compatible with previous observations of Kouba et al. that RNAP, MsHelR and $\sigma^{\mathrm{A}}$ can coexist within

304 one complex (Kouba et al., 2020). The ability of MabHelR to dissociate RNAP trapped in the

305 presence of RIF on the sinP3 promoter DNA, as well as alleviation of transcription inhibition by RIF in

306 multiple round in vitro transcription assays provide support for this model (Figures $3 d$ and $5 c, d$ ). Since 
307 the RIF-stalled complexes constitute significant roadblocks for the transcription-translation machinery

308 and replication, the clearance of these obstacles is imperative for bacterial survival offering a possible

309 explanation for the RIF hypersensitivity of the $\Delta$ helR mutant (Figure 2).

310 Interestingly, data in Figure 5d demonstrate that MabHeIR can dissociate RIF-stalled RNAP in

311 an NTP independent manner and is consistent with previous in vitro studies that describe an NTP

312 independent disassembly of stalled elongation complexes by MsHelR (Kouba et al., 2020).

313 Nevertheless, MabHeIR mutants defective in ATP hydrolysis are also defective in conferring rifamycin

314 resistance implying that ATP hydrolysis must be required for RIF resistance in a step other than

315 disassembly of the RNAP complex (Figure 4). Additionally, removal of the tip of the PCh-loop

316 (MabHelR486-505), specifically mutations in MabHelRE496 and D497 that are in close proximity to

317 RIF ( within $3.6 \AA$ ), abrogates the ability of MabHelR to restore rifamycin resistance of a $\Delta$ Mab_helR

318 mutant but does not affect its ability to dissociate stalled RNAP (Figure 5d). Taken together these data

319 suggest that disruption of stalled RNAP by MabHeIR is insufficient for HelR mediated RIF resistance.

320 We therefore envision that MabHelR may actively displace RIF bound to RNAP following dissociation

321 of the stalled initiation complex (Figure 6, Scenario 1) and is corroborated by studies on the

322 Streptomyces venezuelae HelR which demonstrate displacement of RIF from RNAP using a RIF

323 photoprobe (Gerard Wright, personal communication). The requirement of ATPase activity of

324 MabHelR in RIF resistance suggests that displacement of RIF and/or dissociation of HelR from RNAP

325 may be dependent on ATP hydrolysis. An alternate pathway (Figure 6, scenario 2) could involve

326 removal of RIF by HeIR binding to RIF-trapped initiation complexes, dissociation of HelR and

327 resumption of transcription. This model is however unlikely due to the instability of the RNAP-DNA

328 complex induced by HelR binding. Finally, we cannot rule out that free RNAP bound to RIF could also

329 serve as substrates for HelR, and displacement of RIF by HeIR makes RNAP available for further

330 rounds of transcription (Figure 6, complex B).

331 The function of HelR in RIF resistance, while unprecedented for RNAP, displays a startling

332 resemblance to $\mathrm{HflX}$ and the ribosome protection proteins that confer resistance to ribosome targeting

333 antibiotics. Recently we demonstrated that MabHflX confers resistance to macrolide/lincosamide 
334 antibiotics by dissociating ribosomes stalled in the presence of these drugs (Rudra et al., 2020). A

335 similar mechanism of $\mathrm{HflX}$ mediated macrolide/lincosamide resistance has also been suggested for

336 Listeria monocytogenes (Duval et al., 2018). The clearance of antibiotic stalled macromolecular

337 complexes therefore appears to be a common but underappreciated theme of resistance mechanisms

338 employed by bacteria. Additionally, ribosome protection proteins such as TetM/TetO involved in

339 tetracycline resistance, and the ARE-ABCF proteins involved in resistance to antibiotics that bind the

340 peptidyl transferase center/ nascent polypeptide exit tunnel act by displacing the ribosome bound

341 antibiotic (Connell et al., 2003; Sharkey et al., 2016). The HelR mediated rifamycin resistance

342 mechanism therefore appears to integrate the functions of both $\mathrm{HflX}$ and the ribosome protection

343 proteins within a single protein.

344 The inducible expression of Mab_arr and HelR renders RIF completely ineffective as a

345 therapeutic option against M. abscessus infections. RBT induced expression of Mab_arr and

346 Mab_helR could potentially limit the efficacy of RBT treatment as well. Interestingly, the presence of

347 the conserved 19-bp inverted repeat RAE sequence identified upstream of both Mab_arr and

348 Mab_helR suggests the presence of a common regulator. Since a double mutant is more sensitive

349 than a single mutant of either Mab_arr or Mab_helR alone, targeting the common regulator can

350 potentially reduce RIF sensitivity dramatically and reclaim the use of this well tolerated antibiotic.

351 Future characterization of RAE-dependent regulation of Mab_arr and Mab_helR will be of paramount

352 importance as it will enable development of inhibitors that can concomitantly prevent expression of

353 both rifamycin resistance determinants leading to an effective therapeutic regimen containing a

354 rifamycin antibiotic. 


\section{MATERIALS \& METHODS}

\section{Bacterial Strains, Media and Plasmids:}

M. abscessus ATCC19977 strains were grown at $37^{\circ} \mathrm{C}$ in Middlebrook $7 \mathrm{H} 9$ (DIFCO)

359 supplemented with $0.05 \%$ Tween 20 and $10 \%$ OADC/ADC. Gene replacement mutants as well as

360 tagged strains were constructed using recombineering and confirmed by sequencing (Hurst-Hess et

361 al., 2017). Complementing strains were created by cloning WT or mutant HelR in pMH94 under the

362 control of its native promoter followed by integration at the phage L5 attB site. Wt and mutant He/R

363 genes were cloned in pET21a or pET21a-FLAG for overexpression in E.coli. were Deletions and

364 point mutations in HelR were created using the Q5 site directed mutagenesis kit (NEB). Primers for

365 mutant construction were designed using the NEBaseChanger as recommended. All mutants were

366 confirmed by Sanger sequencing. All bacterial strains and plasmids are listed in Table S2 .

Genomes of 9 clinical strains $M$. abascessus were sequenced using the Illumina platform and

assembled using Unicycler v0.4.9b with default parameters. The average nucleotide identities were

calculated from the assemblies using FastANI version 1.32 with default parameters. The reference

genome of M. Abscessus strain ATCC 199977 was retrieved from NCBI under the accession number

NZ_MLCG01000010.1.

Mycobacterium tuberculosis $m c^{2} 7000$, an attenuated strain of Mycobacterium tuberculosis,

373 H37Rv, which carries deletions in RD1 and panCD loci, both of which are critical for virulence of $M$.

374 tuberculosis, was grown at $37^{\circ} \mathrm{C}$ in Middlebrook $7 \mathrm{H} 9$ (DIFCO) supplemented with $10 \%$ OADC and

$375 \quad 0.05 \%$ Tween 20 (Ojha et al., 2008).

\section{Antibiotic Sensitivity Assays}

378 Wild type and mutant strains of $M$. abscessus were grown to an $A_{600}$ of 0.6-0.7. Cells were

379 tested for their susceptibility to rifampicin and rifabutin by spotting a 10- fold serial dilution on

380 Middlebrook 7H10 (DIFCO) plates containing the indicated concentration of antibiotics. Antibiotic

381 susceptibility in liquid media was assayed by inoculating the desired strain in a two-fold dilution series 
382 of each antibiotic at an initial $A_{600}$ of 0.0004 . The cultures were incubated at $37^{\circ} \mathrm{C}$ and the $A_{600}$ was

383 measured after 72 hours.

\section{RNA preparation / qPCR}

M. abscessus (ATCC 19977) was grown to exponential phase (OD=0.4) in Middlebrook 7H9-

OADC and exposed to a sublethal dose $(16 \mu \mathrm{g} / \mathrm{ml})$ of RIF for 30 mins. Total RNA was prepared using

the Qiagen RNA preparation kit followed by DNAse I treatment. Unexposed cells were used as

controls. Approximately $5 \mu \mathrm{g}$ total RNA samples were treated with the Ribo-Zero ${ }^{\mathrm{TM}}$ rRNA removal

procedure (Illumina) to enrich for mRNA. Approximately 500 ng of RNA was used for library

preparation using the Script Seq v2 RNA-Seq kit and high throughput sequencing on the Illumina

NextSeq platform. The sequence data was analyzed using the reference-based analysis and default

parameters on Rockhopper v2.03 in which the data is normalized by upper quartile normalization and

transcript abundance is reported as RPKM. Differential gene expression is tested for each transcript and q-values are then reported that control the false discovery rate (McClure et al., 2013; Tjaden,

2015). RNAseq experiments were performed three independent times, using two biological replicates

\section{7 each time.}

398 M. abscessus strains (ATCC 19977, clinical strains and $\Delta M a b \_h e l R$ ) were grown to $\mathrm{A}_{600}$ of 0.7 ,

exposed to RIF $(8 \mu \mathrm{g} / \mathrm{ml})$ or RBT $(0.5 \mu \mathrm{g} / \mathrm{ml})$ for 30 mins. Total RNA was prepared using the Qiagen

RNA preparation kit followed by DNAse I treatment. Primers for qRT-PCR were generated using

Primer Quest software (IDT). cDNA was generated using random hexamers and Maxima reverse

402 transcriptase (Fisher Scientific), and qRT-PCR performed using the Maxima SYBR Green qPCR

403 Master Mix (Fisher Scientific) using the following primer pairs: Mab_arr 5'-CGTACTTCCATGGCAC

404 CAA -3'/5'-GAATTTCTTGTCCGTCACGTTG-3'and HeIR 5'- GGAGACGAACGTGgTGTTTA-3'/5'-

405 TCGATCACAATGTGTCCATAGG . Applied Biosystems 7300 Real-Time PCR System was used with

406 cycling conditions of: $50^{\circ} \mathrm{C}$ for $2 \mathrm{~min}, 95^{\circ} \mathrm{C}$ for $10 \mathrm{~min}$, and 40 cycles of $95^{\circ} \mathrm{C}$ for $15 \mathrm{~s}, 60^{\circ} \mathrm{C}$ for $1 \mathrm{~min}$.

407 Data represents mean $\pm S D, n=3$. 


\section{Protein overexpression and purification}

Wild type and mutant heIR were cloned into pET21a with either a C-terminal his-tag or a C-

411 terminal FLAG tag, transformed into BL21(DE3) pLysS, grown to an $\mathrm{A}_{600}$ of 0.5 and induced with $1 \mathrm{mM}$

412 IPTG at $30^{\circ} \mathrm{C}$. The cells were lysed in a buffer containing $50 \mathrm{mM}$ Tris- $\mathrm{HCl}(\mathrm{pH} 8.0), 300 \mathrm{mM} \mathrm{NaCl}$,

$4131 \mathrm{mM} \mathrm{MgCl} 2,5 \%$ glycerol, $1 \mathrm{mM} \mathrm{PMSF}$ and $0.25 \%$ sodium deoxycholate and the clarified lysate was

414 loaded on a Ni-NTA column (Qiagen). Non-specifically bound proteins were removed by washing with

415 lysis buffer containing $20 \mathrm{mM}$ imidazole and the proteins eluted with buffer containing $150 \mathrm{mM}$

416 imidazole. For purification of FLAG-tagged proteins, cells were lysed in a buffer containing 50mM Tris-

$417 \mathrm{HCl}(\mathrm{pH} 8.0), 150 \mathrm{mM} \mathrm{NaCl}, 1 \mathrm{mM} \mathrm{MgCl}_{2}, 5 \%$ glycerol and mixed with Anti-FLAG M2 magnetic beads

418 (Sigma). Following wash with the same buffer, FLAG-tagged proteins were eluted by competition

419 using the FLAG peptide (Sigma).

420 M. tuberculosis $\sigma^{\mathrm{A}}$ cloned in pET21a with a C-terminal his -tag was transformed into

421 BL21(DE3) pLysS, grown to an $\mathrm{A}_{600}$ of 0.4 and induced with $1 \mathrm{mM}$ IPTG at $30^{\circ} \mathrm{C}$. The cells were lysed

422 in a buffer containing $50 \mathrm{mM}$ Tris- $\mathrm{HCl}(\mathrm{pH} 8.0), 300 \mathrm{mM} \mathrm{NaCl}$ and $5 \%$ glycerol and the clarified lysate

423 was loaded on a Ni-NTA column (Qiagen). Non-specifically bound proteins were removed by washing

424 with lysis buffer containing $40 \mathrm{mM}$ imidazole and $\sigma^{\mathrm{A}}$ eluted with $150 \mathrm{mM}$ imidazole. For purification of

425 M. tuberculosis RNA polymerase, BL21(DE3) pLysS was co-transformed with pETDuet-Mtb $\beta \beta^{\prime}$ and

426 pRsfDuet-Mtb $\alpha \omega$, grown at $30^{\circ} \mathrm{C}$ to an $\mathrm{A}_{600}$ of 0.4 and induced with $0.4 \mathrm{mM}$ IPTG at $16^{\circ} \mathrm{C}$ for a period

427 of 18 hours. The cells were lysed by sonication and passed through a Ni-NTA column (Qiagen)

428 equilibrated with $50 \mathrm{mM}$ Tris, $300 \mathrm{mM} \mathrm{NaCl}, 1 \mathrm{mM} \mathrm{MgCl}_{2}$, and $5 \%$ glycerol, protease inhibitor cocktail

429 (Lysis buffer). The column was washed Lysis Buffer + 40mM imidazole and eluted with Lysis Buffer

$430+150 \mathrm{mM}$ imidazole. Fractions containing RNAP were loaded on a Heparin Sepharose matrix (GE

431 Healthcare) equilibrated with $50 \mathrm{mM}$ Tris, $300 \mathrm{mM} \mathrm{NaCl}$ and $5 \%$ glycerol and eluted with a buffer

432 containing $1 \mathrm{M} \mathrm{NaCl}$. 
For purification of $M$. abscessus RNA polymerase, MabATCC-rpoA $\mathrm{A}_{\text {is }}$ in which the native $r p o A$

434 was tagged at the $\mathrm{C}$-terminal with $10 \mathrm{X}$-his was grown to an $\mathrm{A}_{600}=0.8$. Cells were treated with

$43516 \mu \mathrm{g} / \mathrm{mL}$ RIF for 30 mins when required. The cells were harvested and lysed using The CryoMill

436 (Retsch) in a buffer containing $50 \mathrm{mM}$ Tris, $200 \mathrm{mM} \mathrm{NaCl}, 5 \%$ glycerol, $1 \mathrm{mM} \mathrm{MgCl} 2$, protease inhibitor

437 cocktail and loaded onto a Ni-NTA matrix equilibrated with the above buffer containing $5 \mathrm{mM}$

438 imidazole. Non-specific proteins were removed by washing with buffer containing 40mM imidazole

439 followed by elution with 150mM imidazole buffer.

\section{Immunoprecipitation and Western Blotting}

MabATCC-helR $R_{\mathrm{FLAG}}$ in which the native helR was tagged at the C-terminal with 3X-FLAG was

443 grown to an $A_{600}=0.8$. Cultures were treated with $8-16 \mu \mathrm{g} / \mathrm{mL}$ RIF for $30 \mathrm{mins}$ and cells were

444 harvested and lysed using The CryoMill (Retsch) in a buffer containing 50mM Tris, 50mM NaCl, 5\%

445 glycerol, $1 \mathrm{mM} \mathrm{MgCl}_{2}$, protease inhibitor. The lysate was clarified by centrifugation, protein

446 concentration was determined at $A_{260}$ and equal quantities of protein from different samples were

447 mixed with Anti-FLAG M2 magnetic beads (Sigma), washed with 20 bed volumes to remove non-

448 specifically bound proteins and eluted using $0.1 \mathrm{M}$ glycine, $\mathrm{pH}$ 3.0. The eluates were neutralized using

$4491 \mathrm{M}$ Tris , $\mathrm{pH}$ 8.0, separated using 8\% SDS-PAGE transferred onto a PVDF membrane and probed

450 with anti -FLAG (Sigma) and anti- RpoA (Biolegend) monoclonal antibodies. Purified Mtb RNAP was

451 used as a control.

452

453 In vitro transcription Assays

$454 \quad$ Multiple round in vitro transcription was performed as previously described (Huang et al.,

455 2012). In short, 75nM M. tuberculosis RNAP was assembled with 300nM purified $\sigma^{\mathrm{A}}$ in a volume of

$45610 \mu \mathrm{l}$ for 10 minutes at $37^{\circ} \mathrm{C}$. RIF was added to indicated concentrations for 15 mins at $37^{\circ} \mathrm{C} .25 \mathrm{nM}$ of

$457 \sin P 3$ promoter DNA was added to the mixtures and incubated for 10 mins at $37^{\circ} \mathrm{C}$. Purified MabHelR

458 was added as indicated. Transcription was initiated by addition of $2 \mu \mathrm{L}$ of NTP mix (1.5mM of ATP, 
459 GTP and CTP and $0.5 \mathrm{mM}$ UTP) $+2 \mu \mathrm{Ci}$ of ${ }^{32} \mathrm{P}-\alpha-\mathrm{UTP}$. Reactions were incubated at $37^{\circ} \mathrm{C}$ for $30 \mathrm{mins}$

460 and terminated by the addition of $5 \mathrm{mM}$ EDTA $+100 \mu \mathrm{g} / \mathrm{ml}$ tRNA. Samples were ethanol precipitated,

461 resuspended in STOP buffer (80\% v/v Formamide/10mM EDTA/0.01\% Xylene Cyanol/0.01\%

462 Bromophenol Blue) and separated using denaturing PAGE (6\% Urea polyacrylamide gel). The

463 products were visualized using a Typhoon Imager (GE Healthcare) and quantitated using the Image

464 Quant software.

\section{Generation of RIF-stalled RNAP complexes and disassembly}

RNAP stalled in the presence of RIF was generated by using a modification of a previous

protocol for trapping stalled elongation complexes (Kouba et al., 2020). First, holoenzyme was

for 5 mins followed by addition of 90 pmoles of RIF and $1 \mathrm{mM} \mathrm{ATP} \mathrm{for} 10$ mins at $25^{\circ} \mathrm{C}$ to enable

formation of stalled RNAP complexes. Streptavidin coated magnetic beads (Pierce, $25 \mu \mathrm{l}$ per sample)

was washed with $500 \mu$ l of binding buffer $(20 \mathrm{mM}$ Tris, $\mathrm{pH} 8.0,100 \mathrm{mM} \mathrm{NaCl})$, resuspended in $25 \mu \mathrm{l}$ of

the same buffer. The assembled stalled complexes were mixed with washed buffer and incubated at

$2 \mathrm{mM} \mathrm{MgCl}_{2}$ and $\left.1 \mathrm{mM} \mathrm{DTT}\right)$ followed by a wash with $500 \mu$ reaction buffer (40mM Tris, $\mathrm{pH} 8.0,10 \mathrm{mM}$

$\mathrm{MgCl}_{2}, 100 \mathrm{mM} \mathrm{KCl}$ and $\left.1 \mathrm{mM} \mathrm{DTT}\right)$. The beads were resuspended in $10 \mu$ reaction buffer containing

WT or mPCh mutant of HelR with or without ATP $(1 \mathrm{mM})$ and incubated at $37^{\circ} \mathrm{C}$ for 30 mins. The 


\section{ATP hydrolysis assay}

486 ATPase activity of Wt and mutant HelR proteins was determined using the colorimetric ATPase assay

487 kit (Sigma). The ATP hydrolysis reaction was performed by incubating $0.3 \mu \mathrm{M}$ HelR with 1 mM ATP

488 (NEB), in a buffer containing $40 \mathrm{mM}$ Tris, $80 \mathrm{mM} \mathrm{NaCl}, 8 \mathrm{mM}$ MgAc2, 1 mM EDTA, pH 7.5 for 30mins

489 at room temperature followed by addition of the malachite green reagent included in the kit. Following

490 incubation for 30 mins at room temperature to generate the colorimetric product, the absorbance of

491 the samples was measured at $620 \mathrm{~nm}$. The amount of free phosphate liberated was determined from a

492 standard curve that was generated using phosphate standards provided in the kit.

493

\section{Protein identification through mass spectrometry}

495 LC MS/MS was performed by the RNA Epitranscriptomics \& Proteomics Resource at the

496 University at Albany. Briefly, the protein band corresponding in size to MabHelR in RNAP

497 preparations was manually excised, minced, in-gel digested with trypsin (Sigma, St. Louis, MO) and

498 extracted three times with $50 \%$ acetonitrile containing $5 \%$ formic acid. LC-MS/MS was performed on

499 an integrated micro LC-Orbitrap Velos system (Thermo). Tandem spectrum data was processed using

500 Mascot 2.7 (Matrix Science, Boston, MA). The list of peaks was used to query the Mycobacteriaceae

501 protein database downloaded from NCBI by setting the following parameters: peptide mass tolerance,

$50210 \mathrm{ppm}$; MS/MS ion mass tolerance, $0.1 \mathrm{Da}$; allowing up to one missed cleavage; considering variable

503 modifications such as methionine oxidation, cysteine carboxyamidomethylation, and deamidation.

504 Only significant hits as defined by Mascot probability analysis was considered for a positive protein 505 identification. 


\section{Acknowledgements}

508 We thank The Wadsworth Center's Applied Genomics Technology Core, the Bioinformatics Core and

509 the Media Core. We thank Qishan Lin from The University at Albany for performing LC MS/MS for

510 protein identification. We also thank Jon Paczkowski and Anil Ojha for invaluable discussions and

511 critical reading of the manuscript. PG is supported by NIH awards Al155473 and Al146774 and the

512 Wadsworth Center. 


\section{$513 \quad$ Figure Legends}

Figure 1. Exposure of $M$. abscessus ATCC and clinical strains to rifamycins results in

\section{6 induction of MAB_3189c/ helR expression.}

517 a) Volcano plot of differentially expressed genes in M. abscessus ATCC19977 upon exposure to RIF

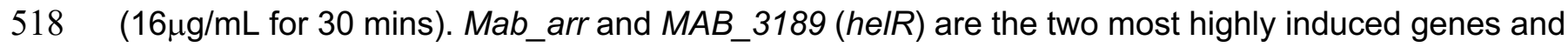

519 are indicated.

520 b) Wild type $M$. abscessus was grown to $A_{600}$ of 0.7 and exposed to either $8 \mu \mathrm{g} / \mathrm{mL}$ of RIF or $0.5 \mu \mathrm{g} / \mathrm{mL}$ 521 of RBT for 30 mins. The amount of Mab_arr and Mab_helR transcript was determined by qPCR and

522 plotted as fold induction over an unexposed control. Data represents mean $\pm \mathrm{SD}, \mathrm{n}=3$. SigA was used

523 as an internal normalization control.

524 c) Average nucleotide identities between eight clinical strains of $M$. abscessus obtained using FastANI 525 version 1.32. is shown.

526 d) Expression of Mab_arr and Mab_helR transcript in eight clinical strains of $M$. abscessus upon

527 exposure to $8 \mu \mathrm{g} / \mathrm{mL}$ of RIF determined by qPCR and plotted as fold induction over an unexposed

528 control. Data represents mean \pm SD, $n=3$. SigA was used as an internal normalization control.

530 Figure 2. Deletion of Mab_helR results in RIF and RBT hypersensitivity in M. abscessus and 531 heterologous expression of $H e I R$ in $M$. tuberculosis increases RIF tolerance.

532 a-b) Growth of ten-fold serial dilutions of $M$. abscessus ATCC 19977, $\triangle M a b \_h e l R, \Delta M a b \_a r r$,

$533 \Delta$ Mab_helR/ $\triangle M a b \_a r r$, and the indicated complementing strains on Middlebrook 7H10 OADC

534 containing indicated concentrations of RIF and RBT. Data is representative of $>3$ independent 535 experiments.

536 c) Real time PCR showing the induction over an unexposed control of Mab_arr transcript in wild type

537 M. abscessus and the $\Delta$ helR strains exposed to either $8 \mu \mathrm{g} / \mathrm{mL}$ of RIF or $0.5 \mu \mathrm{g} / \mathrm{mL}$ of RBT. Data

538 represents mean $\pm \mathrm{SD}, \mathrm{n}=3$. SigA was used as an internal normalization control. 
d) Growth of ten-fold serial dilutions of wild-type M. tuberculosis $m c^{2} 7000, m c^{2} 7000$ containing a

540 chromosomally integrated copy of helR and $m c^{2} 7000$ containing a chromosomally integrated copy of

541 the empty vector (control) on Middlebrook $7 \mathrm{H} 10$ OADC/pan 50ng/mL of RIF. Data is representative of

5422 independent replicates.

543 e) Sequence logo of the19bp inverted repeat sequence (RAE) and alignment of upstream sequences

544 of Mab_helR (MAB_3189), Mab_arr, Ms_helR (MSMEG_2174) and Ms_arr. Region of RAE deleted is

545 shaded in grey.

546 f) Growth of ten-fold serial dilutions of $M$. abscessus ATCC 19977, $\triangle M a b \_$helR and complementing

547 strains containing either helR expressed from a native promoter or helR expressed from a promoter

548 lacking one half of RAE on Middlebrook $7 \mathrm{H} 11$ OADC containing RIF $(16 \mu \mathrm{g} / \mathrm{mL})$.

Figure 3. MabHelR associates with RNAP in vivo and in vitro and rescues inhibition of in vitro transcription in the presence of RIF.

552 a) Silver stained gel showing RNAP purified from untreated and RIF treated $(16 \mu \mathrm{g} / \mathrm{mL}$ of RIF for 30

553 mins) M. abscessus strain in which rpoA was C-terminally tagged with the 10X-his epitope at its native

554 chromosomal location. Purified MabHelR is used as a control. RNAP purified from RIF treated

555 samples was loaded on a preparative SDS PAGE, Coomassie stained and a band corresponding in

556 size to MabHelR was excised and analyzed by LC MS/MS.

557 b) An $M$. abscessus strain in which helR was C-terminally tagged with the 3X-FLAG epitope at its 558 native chromosomal location was either untreated or treated with $8-16 \mu \mathrm{g} / \mathrm{mL}$ of RIF for 30 mins. Wild-

559 type M. abscessus with an untagged helR gene was used as a control. Samples were normalized by

560 wet cell weight and FLAG tagged proteins were purified using anti-FLAG M2 beads. Samples were

561 analyzed by immunoblotting using anti-FLAG and anti-RpoA antibodies. Purified RNAP was used as a

562 marker. Data is representative of $>3$ biological replicates.

563 c) Coomassie stained gel showing elution of proteins using nickel affinity chromatography. Interaction

564 assays were carried out using HelR FLAG $_{\text {and }}$ RNAP $_{\text {his }}$ followed by binding and elution from a Ni-NTA 
565 column. Control samples included either only RNAP his $_{\text {or HelR }}$ FLAG. Purified RNAP his or HelR FLAG $_{\text {are }}$ 566 included as markers.

567 d) Multiple-round in vitro transcription assays were performed on the $\sin P 3$ promoter using $75 n M \sigma^{\mathrm{A}}-$

568 RNAP. RIF was added to indicated concentrations for 10 mins at $37^{\circ} \mathrm{C}$ followed by addition of a 9 - or

569 15-fold molar excess of MabHelR. Transcription was initiated by addition of NTPs and incubated at

$57037^{\circ} \mathrm{C}$ for 30 mins. Samples were separated using denaturing PAGE (6\% Urea polyacrylamide gel)

571 and the 77nt product was visualized using a Typhoon Imager (GE Healthcare) and quantitated using

572 the Image Quant software. The inhibition of transcription at each RIF concentration is expressed as a

573 ratio of the activity in the absence and presence of RIF.

575 Figure 4. MabHeIR is an ATPase and ATP hydrolysis is required for conferring RIF/RBT

576 resistance.

577 a) Domain organization of MabHelR showing the location of Walker A and Walker B motifs within

578 domains $1 \mathrm{~A} 1$ and $1 \mathrm{~A} 2$. The strictly conserved residues in each motif are underlined and the residues

579 mutated to alanine in the study are in red.

580 b) ATPase activity of Wt MabHeIR is shown in the presence and absence of MabRNAP. MabHelR

581 mutations in either Walker B alone (mWalkerB) or Walker A and B (mWalkerAB) are defective in ATP

582 hydrolysis. Data represents mean $\pm \mathrm{SD}, \mathrm{n}=3$

583 c) Growth of ten-fold serial dilutions of $M$. abscessus ATCC 19977, $\triangle M a b \_h e I R$ and $\Delta M a b \_h e l R$

584 complemented with either WT helR, helR (mWalkerB) or helR (mWalkerAB) on Middlebrook 7H10-

585 OADC containing RIF $(16 \mu \mathrm{g} / \mathrm{mL})$ or $\operatorname{RBT}(1.5 \mu \mathrm{g} / \mathrm{mL})$. Data is representative of at least 3 independent 586 experiments.

588 Figure 5. PCh loop of MabHeIR is essential for conferring RIF/RBT resistance.

589 a) Location of the MabHelR PCh loop (orange) containing helices $\alpha 16$ and $\alpha 17$ relative to the active 590 site (AS) residues (gray) in $\beta^{\prime}$ (yellow) and RIF (blue) is shown. Structure of MabHeIR was modeled 
using Phyre and superimposed on the structure of MsHelR bound to RNAP (PDB ID 6YYS) in

592 PyMOL v2.3. 0. The location of RIF was determined from the structure of RIF bound M. smegmatis

593 RNAP (PDB ID 6CCV). Black arrows show the regions of MabHelR PCh loop that are deleted in

594 MabHelR $\triangle \mathrm{PCh} 1$ and magenta arrows show the deleted regions in MabHelR $\Delta \mathrm{PCh}$. Enlarged box

595 below shows the location of the 494-DDED-497 motif at the tip of the PCh loop and the proximity of

596 E496/ D497 to RIF.

597 b) Growth of ten-fold serial dilutions of $M$. abscessus ATCC 19977, $\Delta M a b \_h e I R$ and $\Delta M a b \_h e l R$

598 complemented with either WT helR, helR ( $\triangle \mathrm{PCh} 1)$ heIR ( $\triangle \mathrm{PCh} 2)$ and helR (mPCh) on Middlebrook

$5997 \mathrm{H} 10-\mathrm{OADC}$ containing RIF $(16 \mu \mathrm{g} / \mathrm{mL})$ or RBT $(1.5 \mu \mathrm{g} / \mathrm{mL})$. Data is representative of at least 3

600 independent experiments. $\triangle \mathrm{PCh} 1$ corresponds to deletion of residues $467-515, \triangle \mathrm{PCh} 2$ : deletion of

601 residues 486-505 and mPCh corresponds to point mutations E496A/D496A.

602 c) Schematic representation of experimental design. RNAP stalling on 5'-bio sinP3 DNA was carried

603 out in the presence of RIF and ATP, the +1 nucleotide. The stalled complexes were tethered on

604 streptavidin magnetic beads. Upon addition of MabHelR, the dissociated RNAP is expected to be

605 present in the eluate and RNAP bound to DNA remains tethered on the beads.

606 d) Western blot of samples eluted from streptavidin magnetic beads tethered to DNA with RIF trapped

607 RNAP complexes using anti-his antibodies that recognize his-tagged RpoA. Western blots are

608 quantitated using ImageJ. Data represents mean \pm SD, $n=3$.

610 Figure 6. Model of HeIR mediated RIF resistance. Two species of RNAP that can serve as

611 substrates for HelR and are labeled as (A) RNAP stalled during initiation in the presence of RIF and (

612 B) free RNAP. Possible pathways for HelR action leading to RNAP recycling are illustrated.

613 
614 Table 1. Survival of wild type M. abscessus ATCC19977, $\Delta H e l R, \Delta M a b \_a r r, \Delta M a b \_h e l R /$

$615 \Delta M a b \_a r r, \Delta h e I R+p M H 94-h e / R$ in a 2-fold dilution series of RIF and RBT in Middlebrook 7H9/OADC

616 medium. The minimum concentration of antibiotic required to inhibit $99 \%$ of growth after 72 hours is

617 shown. Data is representative of 3 replicates. 


\section{Supplementary Figures:}

619 Figure S1: a)Wild type $M$. abscessus, $\Delta$ helR and complemented strains were grown to $A_{600}$ of 0.7 and

620 a 10-fold dilution series was spotted on media lacking antibiotics. No growth defect was observed in

621 the $\Delta$ helR mutant compared to WT bacteria. b) Wild type M. smegmatis, $\Delta M s \_h e l R$ and $\Delta M s \_h e / R$

622 complemented with either HelR or Ms_helR were grown to $\mathrm{A}_{600}$ of 0.7 and a 10 -fold dilution series

623 was spotted on media RIF $(16 \mu \mathrm{g} / \mathrm{mL})$.

Figure S2: Sequence comparison of HelR from M. abscessus and M. smegmatis. Sequence

626 alignment was performed using CLUSTALW. The N-terminal, C-terminal, $1 \mathrm{~A}-1$ and $1 \mathrm{~A}-2$ and the HeIR 627 specific domains are shaded.

629 Figure S3: Expression of mutant HeIR in vivo and interaction with RNAP in vitro. b) Immunoblot 630 with anti-FLAG antibodies of $M$. abscessus ATCC 19977, $\Delta$ Mab_helR and $\Delta$ Mab_helR complemented

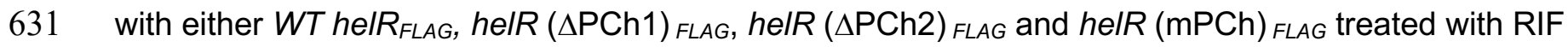

$632(16 \mu \mathrm{g} / \mathrm{mL})$ shows that WT and mutant proteins are induced to similar levels in the complementing 633 strains. c) Coomassie stained gel showing elution of proteins using nickel affinity chromatography.

634 Interaction assays were carried out using WT and mutant HeIR $\mathrm{FLAG}_{\text {with }} \mathrm{RNAP}_{\text {his }}$ as indicated and 635 followed by binding and elution from a Ni-NTA column. Control samples included either only RNAP $_{\text {his }}$

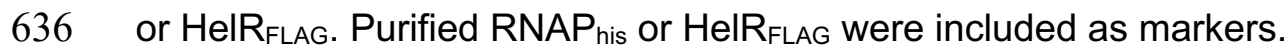




\section{REFERENCES}

642

643

644

645

646

647

648

649

650

651

652

653

654

655

656

657

658

659

660

661

662

663

664

665

666

667

668

669

670

671

672

673

674

675

676

677

678

679

680

681

682

683

684

685

686

687

688
(2013). CF foundation data

Aziz, D.B., Low, J.L., Wu, M.L., Gengenbacher, M., Teo, J.W.P., Dartois, V., and Dick, T. (2017). Rifabutin Is Active against Mycobacterium abscessus Complex. Antimicrob Agents Chemother 61. Ballarino, G.J., Olivier, K.N., Claypool, R.J., Holland, S.M., and Prevots, D.R. (2009). Pulmonary nontuberculous mycobacterial infections: antibiotic treatment and associated costs. Respir Med 103, 1448-1455.

Bittner, M.J., and Preheim, L.C. (2016). Other Slow-Growing Nontuberculous Mycobacteria. Microbiol Spectr 4.

Blaschke, T.F., and Skinner, M.H. (1996). The clinical pharmacokinetics of rifabutin. Clin Infect Dis 22 Suppl 1, S15-21; discussion S21-12.

Brown, B.A., Wallace, R.J., Jr., and Onyi, G.O. (1992a). Activities of clarithromycin against eight slowly growing species of nontuberculous mycobacteria, determined by using a broth microdilution MIC system. Antimicrob Agents Chemother 36, 1987-1990.

Brown, B.A., Wallace, R.J., Jr., Onyi, G.O., De Rosas, V., and Wallace, R.J., 3rd (1992b). Activities of four macrolides, including clarithromycin, against Mycobacterium fortuitum, Mycobacterium chelonae, and M. chelonae-like organisms. Antimicrob Agents Chemother 36, 180-184.

Brown-Elliott, B.A., Nash, K.A., and Wallace, R.J., Jr. (2012). Antimicrobial susceptibility testing, drug resistance mechanisms, and therapy of infections with nontuberculous mycobacteria. Clin Microbiol Rev 25, 545-582.

Brown-Elliott, B.A., and Wallace, R.J., Jr. (2002). Clinical and taxonomic status of pathogenic nonpigmented or late-pigmenting rapidly growing mycobacteria. Clin Microbiol Rev 15, 716-746. Campbell, E.A., Korzheva, N., Mustaev, A., Murakami, K., Nair, S., Goldfarb, A., and Darst, S.A. (2001). Structural mechanism for rifampicin inhibition of bacterial rna polymerase. Cell 104, 901-912. Caruthers, J.M., and McKay, D.B. (2002). Helicase structure and mechanism. Curr Opin Struct Biol $12,123-133$.

Catherinot, E., Roux, A.L., Vibet, M.A., Bellis, G., Ravilly, S., Lemonnier, L., Le Roux, E., BernedeBauduin, C., Le Bourgeois, M., Herrmann, J.L., et al. (2013). Mycobacterium avium and Mycobacterium abscessus complex target distinct cystic fibrosis patient subpopulations. J Cyst Fibros 12, 74-80.

Connell, S.R., Tracz, D.M., Nierhaus, K.H., and Taylor, D.E. (2003). Ribosomal protection proteins and their mechanism of tetracycline resistance. Antimicrob Agents Chemother 47, 3675-3681. Dey, A., Verma, A.K., and Chatterji, D. (2010). Role of an RNA polymerase interacting protein, MsRbpA, from Mycobacterium smegmatis in phenotypic tolerance to rifampicin. Microbiology (Reading) 156, 873-883.

Dick, T., Shin, S.J., Koh, W.J., Dartois, V., and Gengenbacher, M. (2020). Rifabutin Is Active against Mycobacterium abscessus in Mice. Antimicrob Agents Chemother 64.

Duval, M., Dar, D., Carvalho, F., Rocha, E.P.C., Sorek, R., and Cossart, P. (2018). HflXr, a homolog of a ribosome-splitting factor, mediates antibiotic resistance. Proc Natl Acad Sci U S A 115, 1335913364.

Esteban, J., Garcia-Pedrazuela, M., Munoz-Egea, M.C., and Alcaide, F. (2012). Current treatment of nontuberculous mycobacteriosis: an update. Expert Opin Pharmacother 13, 967-986.

Floto, R.A., Olivier, K.N., Saiman, L., Daley, C.L., Herrmann, J.L., Nick, J.A., Noone, P.G., Bilton, D., Corris, P., Gibson, R.L., et al. (2016). US Cystic Fibrosis Foundation and European Cystic Fibrosis Society consensus recommendations for the management of non-tuberculous mycobacteria in individuals with cystic fibrosis: executive summary. Thorax 71, 88-90.

Ganapathy, U.S., Dartois, V., and Dick, T. (2019). Repositioning rifamycins for Mycobacterium abscessus lung disease. Expert Opin Drug Discov 14, 867-878. 
689 Goldstein, B.P. (2014). Resistance to rifampicin: a review. J Antibiot (Tokyo) 67, 625-630.

690 Griffith, D.E., Aksamit, T., Brown-Elliott, B.A., Catanzaro, A., Daley, C., Gordin, F., Holland, S.M.,

691 Horsburgh, R., Huitt, G., Iademarco, M.F., et al. (2007). An official ATS/IDSA statement: diagnosis,

692 treatment, and prevention of nontuberculous mycobacterial diseases. Am J Respir Crit Care Med 175,

$693 \quad 367-416$.

$694 \mathrm{Hu}$, Y., Morichaud, Z., Chen, S., Leonetti, J.P., and Brodolin, K. (2012). Mycobacterium tuberculosis

695 RbpA protein is a new type of transcriptional activator that stabilizes the sigma A-containing RNA

696 polymerase holoenzyme. Nucleic Acids Res 40, 6547-6557.

697 Huang, C.L., Lin, P.C., Lee, J.Y., and Chang, Y.T. (2012). Hydrothorax following delayed extravascular migration of a totally implantable venous access device in a child. J Pediatr Surg 47, e14.

Hurst-Hess, K., Biswas, R., Yang, Y., Rudra, P., Lasek-Nesselquist, E., and Ghosh, P. (2019). Mycobacterial SigA and SigB Cotranscribe Essential Housekeeping Genes during Exponential Growth. mBio 10. Species-Specific Repertoire of Genes To Confer Extreme Antibiotic Resistance. Antimicrob Agents Chemother 61.

Jacques, J.F., Rodrigue, S., Brzezinski, R., and Gaudreau, L. (2006). A recombinant Mycobacterium tuberculosis in vitro transcription system. FEMS Microbiol Lett 255, 140-147.

Kothavade, R.J., Dhurat, R.S., Mishra, S.N., and Kothavade, U.R. (2013). Clinical and laboratory aspects of the diagnosis and management of cutaneous and subcutaneous infections caused by rapidly growing mycobacteria. Eur J Clin Microbiol Infect Dis 32, 161-188.

Kouba, T., Koval, T., Sudzinova, P., Pospisil, J., Brezovska, B., Hnilicova, J., Sanderova, H., Janouskova, M., Sikova, M., Halada, P., et al. (2020). Mycobacterial HelD is a nucleic acids-clearing factor for RNA polymerase. Nat Commun 11, 6419.

Martiniano, S.L., and Nick, J.A. (2015). Nontuberculous mycobacterial infections in cystic fibrosis. Clin Chest Med 36, 101-115.

Martiniano, S.L., Sontag, M.K., Daley, C.L., Nick, J.A., and Sagel, S.D. (2014). Clinical significance of a first positive nontuberculous mycobacteria culture in cystic fibrosis. Ann Am Thorac Soc 11, 3644.

McClure, R., Balasubramanian, D., Sun, Y., Bobrovskyy, M., Sumby, P., Genco, C.A., Vanderpool, C.K., and Tjaden, B. (2013). Computational analysis of bacterial RNA-Seq data. Nucleic Acids Res $41, \mathrm{e} 140$.

Nakanaga, K., Hoshino, Y., Era, Y., Matsumoto, K., Kanazawa, Y., Tomita, A., Furuta, M., Washizu, M., Makino, M., and Ishii, N. (2011). Multiple cases of cutaneous Mycobacterium massiliense infection in a "hot spa" in Japan. J Clin Microbiol 49, 613-617.

Newell, K.V., Thomas, D.P., Brekasis, D., and Paget, M.S. (2006). The RNA polymerase-binding protein RbpA confers basal levels of rifampicin resistance on Streptomyces coelicolor. Mol Microbiol 60, 687-696.

Newing, T.P., Oakley, A.J., Miller, M., Dawson, C.J., Brown, S.H.J., Bouwer, J.C., Tolun, G., and Lewis, P.J. (2020). Molecular basis for RNA polymerase-dependent transcription complex recycling by the helicase-like motor protein HelD. Nat Commun 11, 6420.

Ojha, A.K., Baughn, A.D., Sambandan, D., Hsu, T., Trivelli, X., Guerardel, Y., Alahari, A., Kremer, L., Jacobs, W.R., Jr., and Hatfull, G.F. (2008). Growth of Mycobacterium tuberculosis biofilms containing free mycolic acids and harbouring drug-tolerant bacteria. Mol Microbiol 69, 164-174. Park, S., Kim, S., Park, E.M., Kim, H., Kwon, O.J., Chang, C.L., Lew, W.J., Park, Y.K., and Koh, W.J. (2008). In vitro antimicrobial susceptibility of Mycobacterium abscessus in Korea. J Korean Med Sci $23,49-52$. 
737 Pei, H.H., Hilal, T., Chen, Z.A., Huang, Y.H., Gao, Y., Said, N., Loll, B., Rappsilber, J., Belogurov,

738 G.A., Artsimovitch, I., et al. (2020). The delta subunit and NTPase HelD institute a two-pronged

739 mechanism for RNA polymerase recycling. Nat Commun 11, 6418.

740 Rominski, A., Roditscheff, A., Selchow, P., Bottger, E.C., and Sander, P. (2017). Intrinsic rifamycin resistance of Mycobacterium abscessus is mediated by ADP-ribosyltransferase MAB_0591. J Antimicrob Chemother 72, 376-384.

Roux, A.L., Catherinot, E., Ripoll, F., Soismier, N., Macheras, E., Ravilly, S., Bellis, G., Vibet, M.A., Le Roux, E., Lemonnier, L., et al. (2009). Multicenter study of prevalence of nontuberculous mycobacteria in patients with cystic fibrosis in france. J Clin Microbiol 47, 4124-4128.

Rudra, P., Hurst-Hess, K.R., Cotten, K.L., Partida-Miranda, A., and Ghosh, P. (2020). Mycobacterial $\mathrm{HflX}$ is a ribosome splitting factor that mediates antibiotic resistance. Proc Natl Acad Sci U S A 117, 629-634.

Schneider, C.A., Rasband, W.S., and Eliceiri, K.W. (2012). NIH Image to ImageJ: 25 years of image analysis. Nat Methods 9, 671-675.

Sharkey, L.K., Edwards, T.A., and O'Neill, A.J. (2016). ABC-F Proteins Mediate Antibiotic Resistance through Ribosomal Protection. mBio 7, e01975.

Spanogiannopoulos, P., Waglechner, N., Koteva, K., and Wright, G.D. (2014). A rifamycin inactivating phosphotransferase family shared by environmental and pathogenic bacteria. Proc Natl Acad Sci U S A 111, 7102-7107.

Swenson, J.M., Wallace, R.J., Jr., Silcox, V.A., and Thornsberry, C. (1985). Antimicrobial susceptibility of five subgroups of Mycobacterium fortuitum and Mycobacterium chelonae. Antimicrob Agents Chemother 28, 807-811.

Tjaden, B. (2015). De novo assembly of bacterial transcriptomes from RNA-seq data. Genome Biol $16,1$.

Tupin, A., Gualtieri, M., Roquet-Baneres, F., Morichaud, Z., Brodolin, K., and Leonetti, J.P. (2010). Resistance to rifampicin: at the crossroads between ecological, genomic and medical concerns. Int J Antimicrob Agents 35, 519-523.

Wallace, R.J., Jr., Brown, B.A., and Onyi, G.O. (1991). Susceptibilities of Mycobacterium fortuitum biovar. fortuitum and the two subgroups of Mycobacterium chelonae to imipenem, cefmetazole, cefoxitin, and amoxicillin-clavulanic acid. Antimicrob Agents Chemother 35, 773-775.

Wiedermannova, J., Sudzinova, P., Koval, T., Rabatinova, A., Sanderova, H., Ramaniuk, O., Rittich, S., Dohnalek, J., Fu, Z., Halada, P., et al. (2014). Characterization of HelD, an interacting partner of RNA polymerase from Bacillus subtilis. Nucleic Acids Res 42, 5151-5163. 


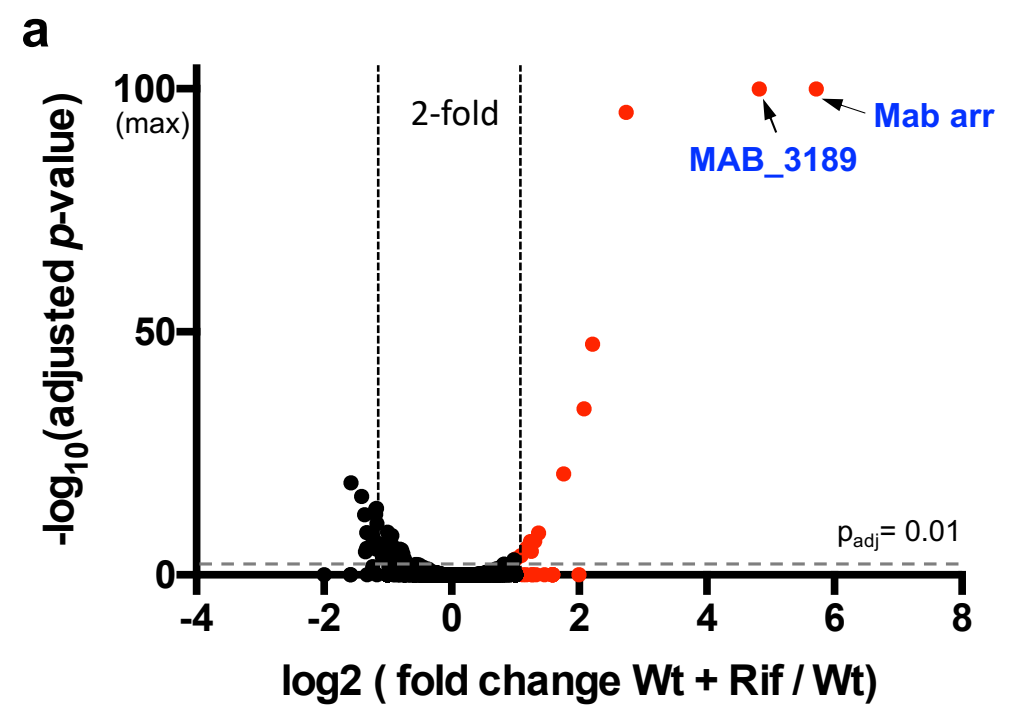

C

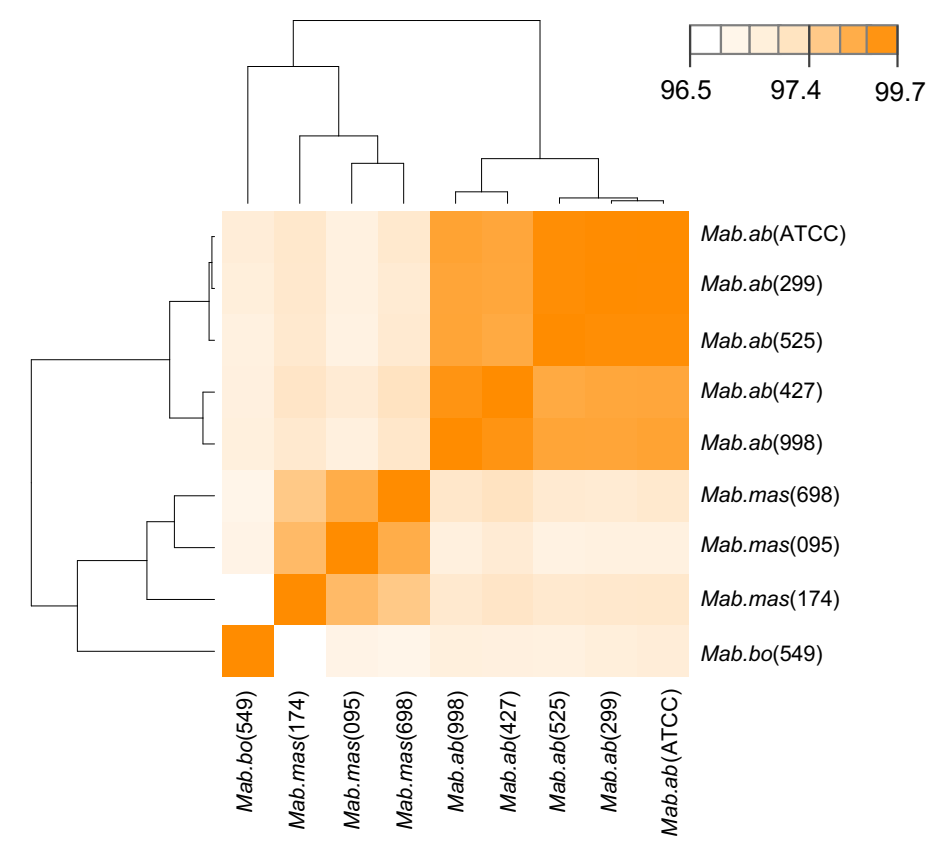

b

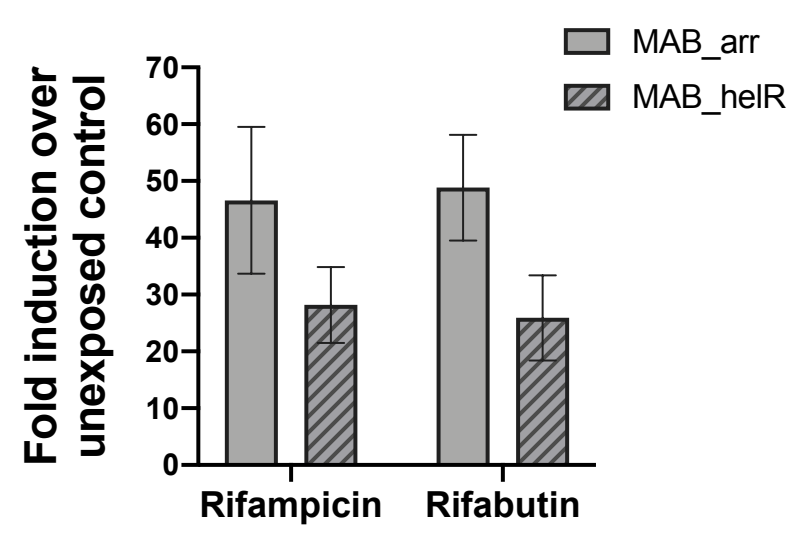


a

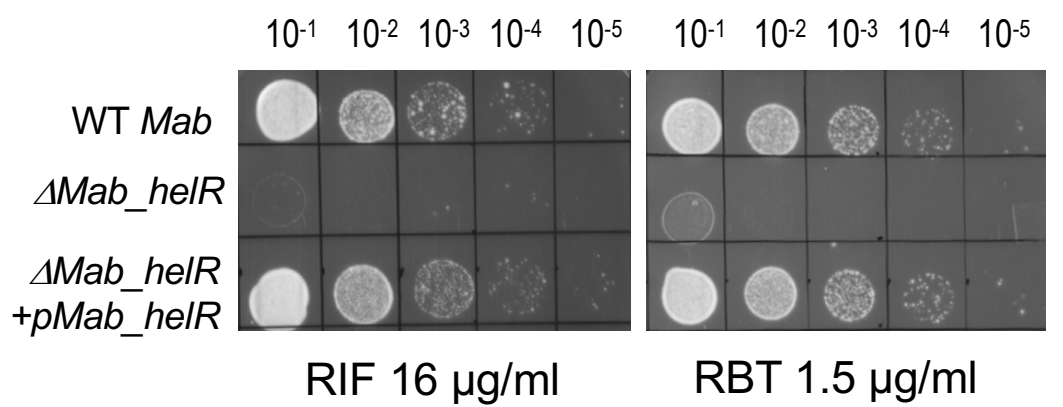

b

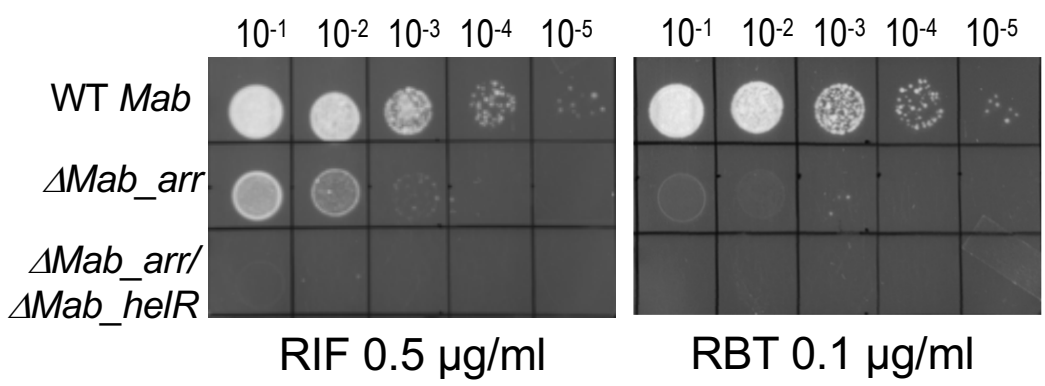

e

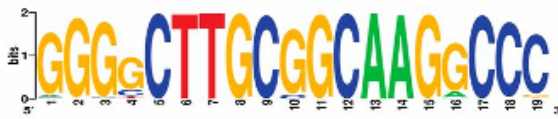

MSMEG_2174 cgccgggaCggGGTCTTGCGGCAAGACCggggtggtggt-[54]- GTG MSMEG Arr gggcgcaggtGGGgCTTGCGGCAAGgCCCggtgccgggt-[54]- GTG MAB_Arrr GTCATGGTGGGGGgCTTGCGGCAAGgCCCCCACCATGAC-[52 ]- ATG MAB_3189c aacgcacaggGGGgCTTGCGGCAAGaCCCgggtgatacc-[57]- ATG
C

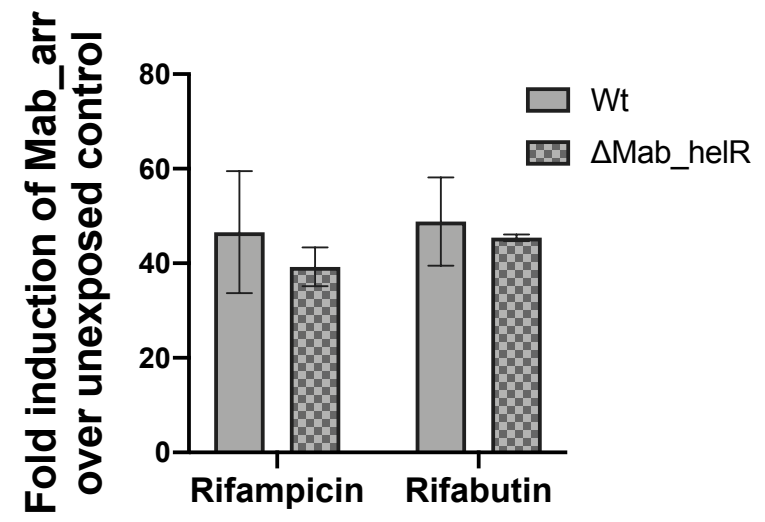

d

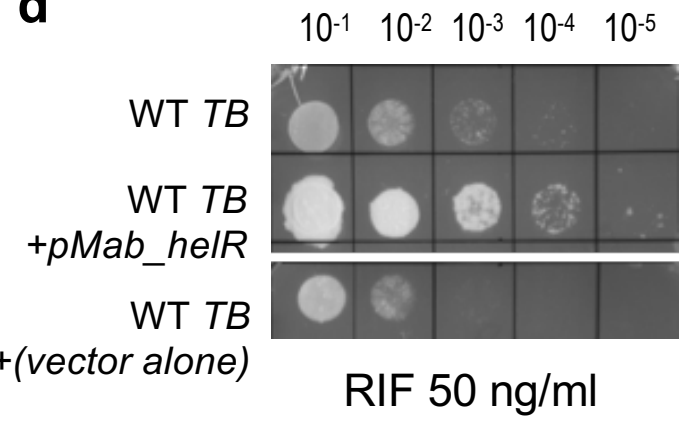

f

$10^{-1} 10^{-2} 10^{-3} \quad 10^{-4} \quad 10^{-5}$

WT Mab

$\triangle M a b \_h e / R$

$\triangle M a b$ helR

$+p M a b \_h e l R$

$\triangle M a b \_h e l R$

$+p M a b$ helR $\triangle R A E$

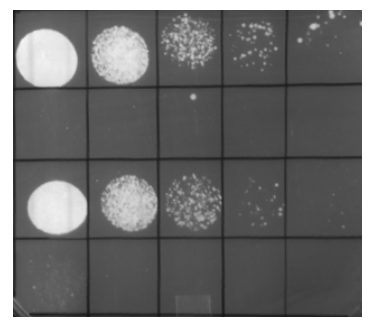

RIF $16 \mu \mathrm{g} / \mathrm{ml}$ 
Purified

a

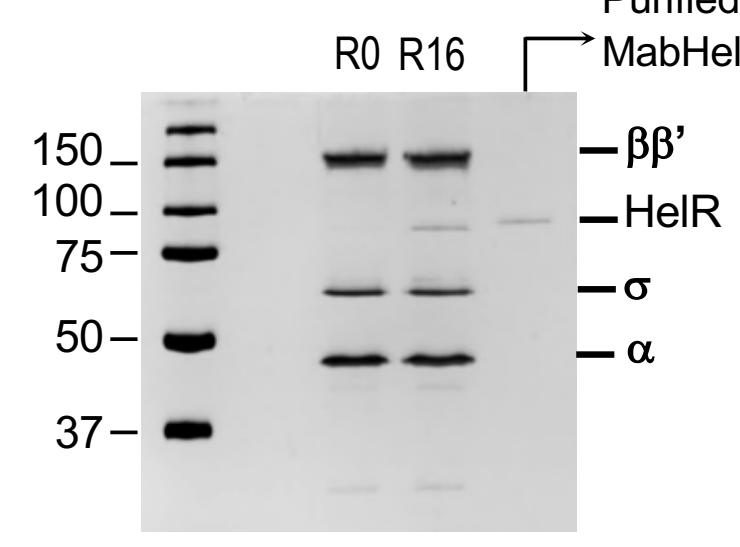

c

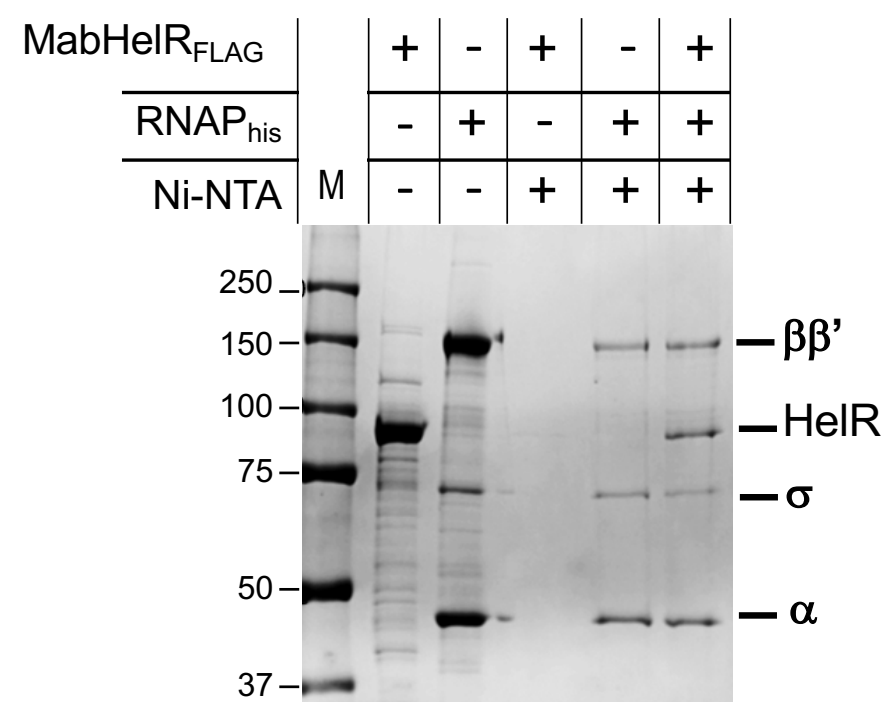

b

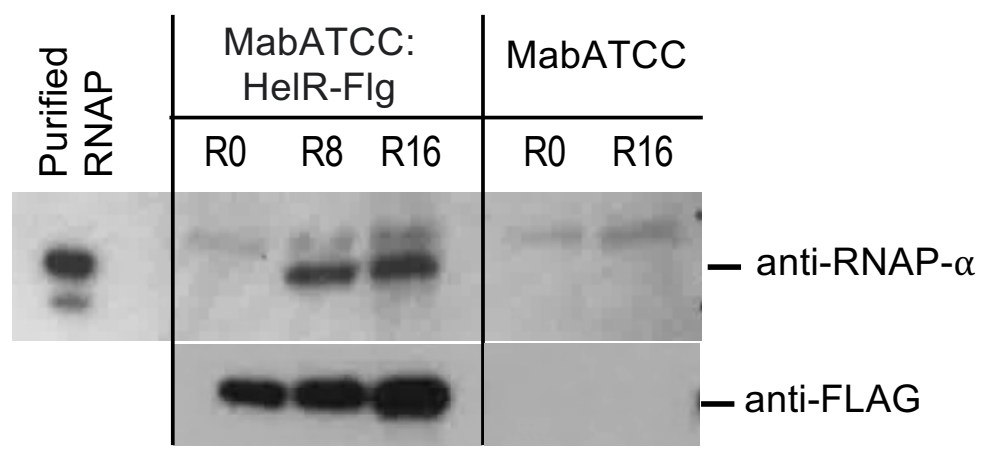

d

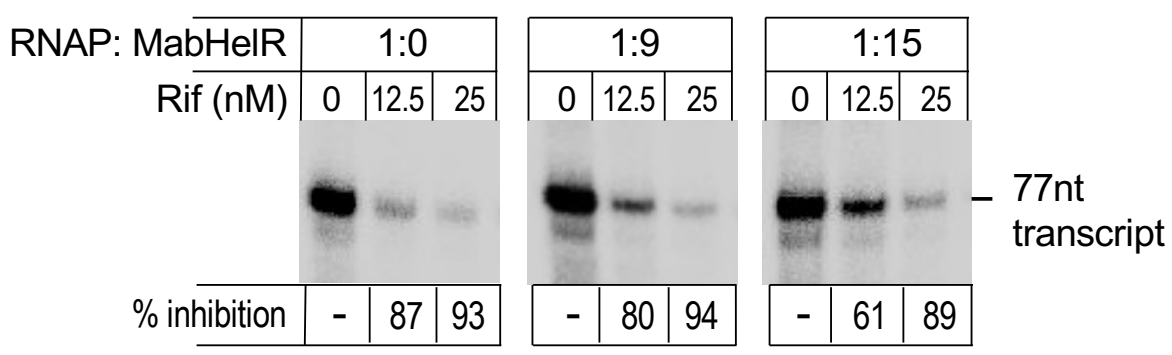

Figure 3 


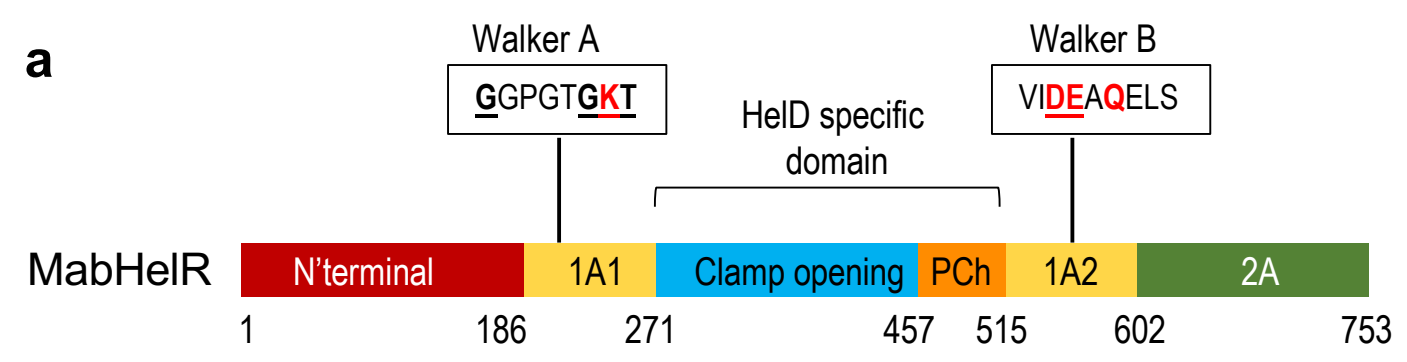

b

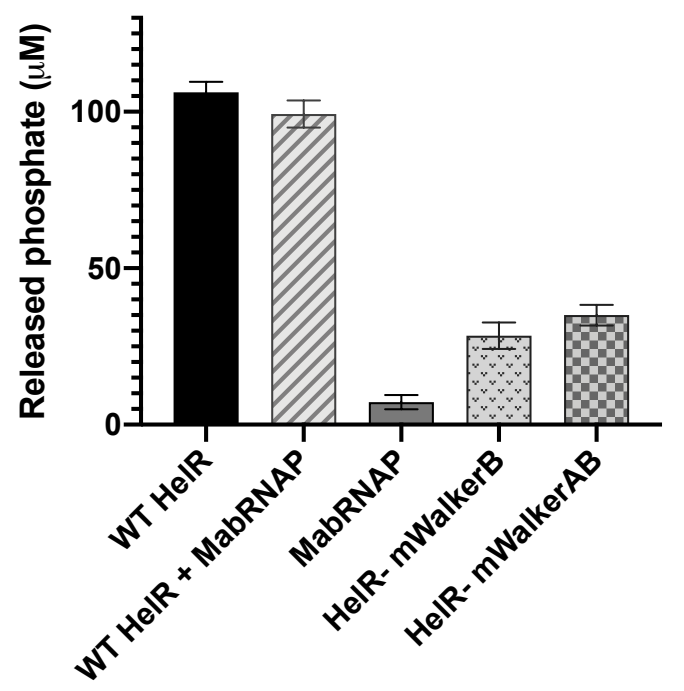

C

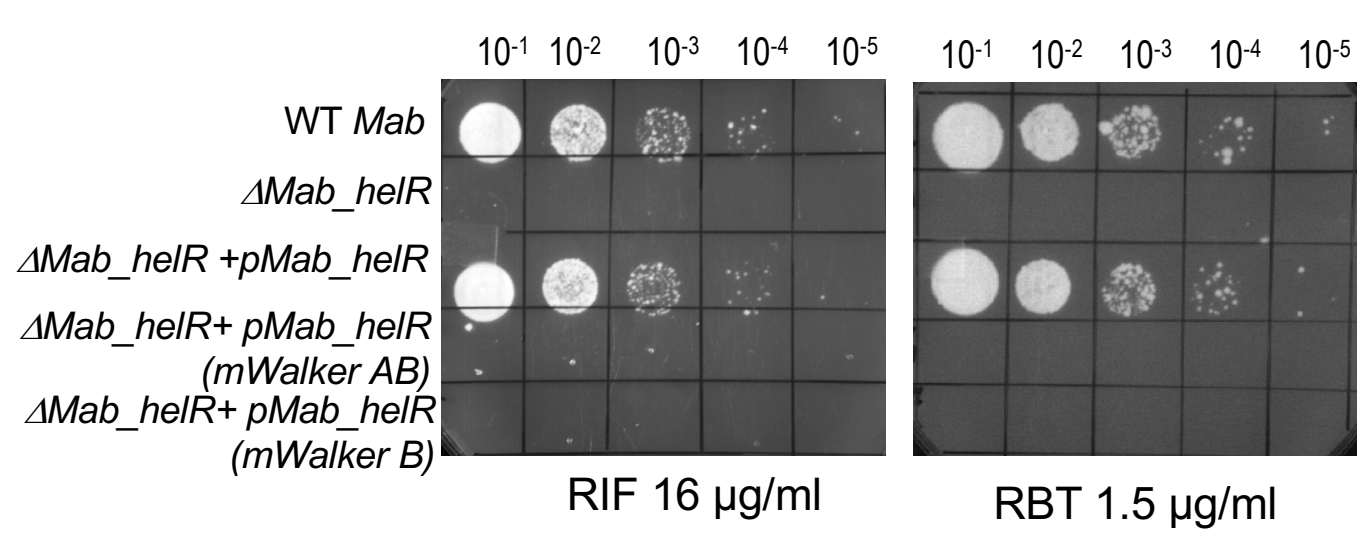

Figure 4 
a

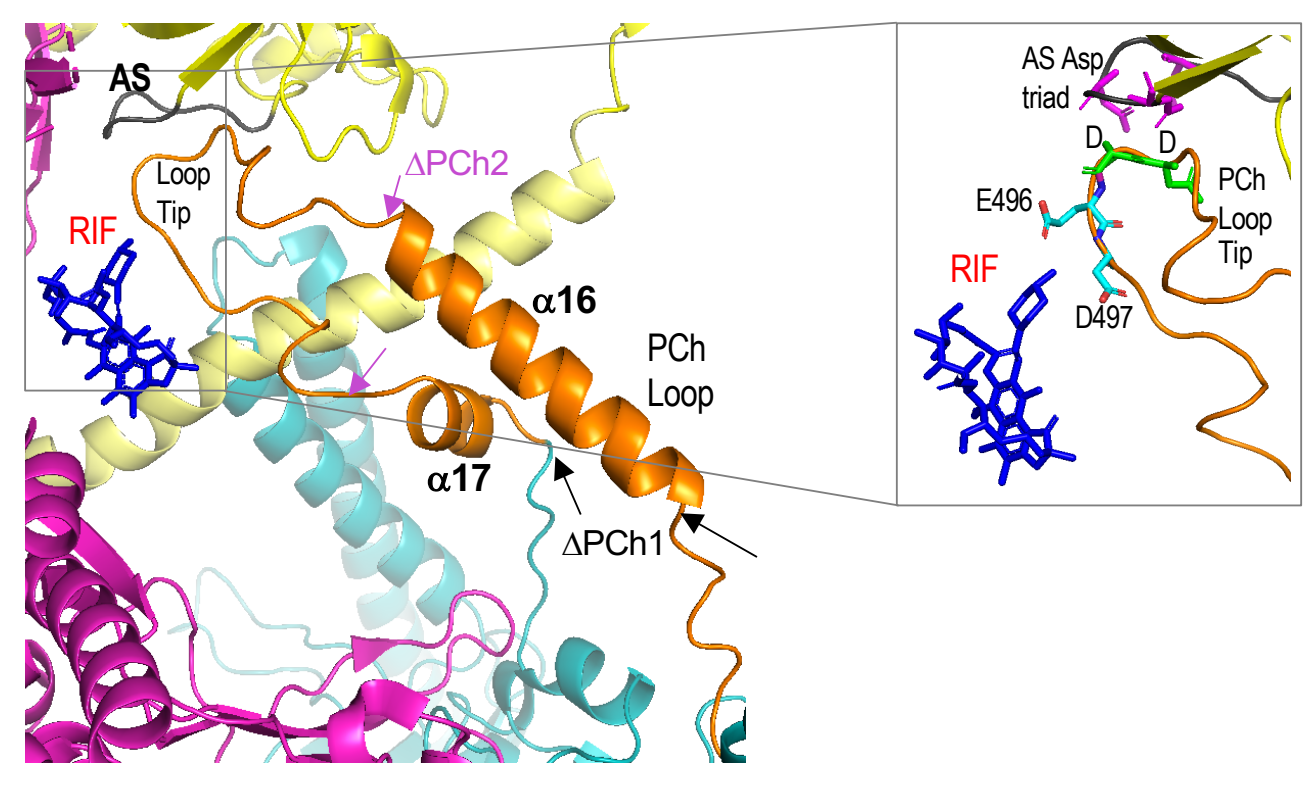

b

b

\section{WT Mab}

$\triangle M a b \_h e l R$

$\triangle M a b \_h e l R$

$+p M a b$ helR $(W T)$

$\triangle M a b$ helR

$+p M a b \_h e l R(\triangle \mathrm{PCh} 1)$

$\triangle M a b$ helR

$+p M a b \_h e l R(\triangle \mathrm{P} c h 2)$

$\triangle M a b$ helR

+pMab_helR(mPCh)

$\begin{array}{llllllllll}10^{-1} & 10^{-2} & 10^{-3} & 10^{-4} & 10^{-5} & 10^{-1} & 10^{-2} & 10^{-3} & 10^{-4} & 10^{-5}\end{array}$

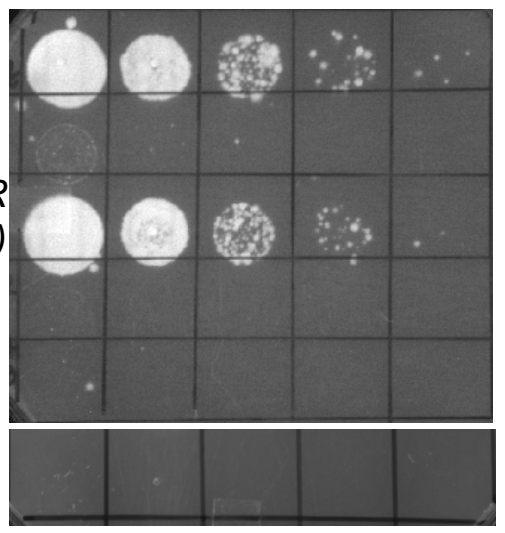

$\mathrm{RIF} 16 \mu \mathrm{g} / \mathrm{ml}$

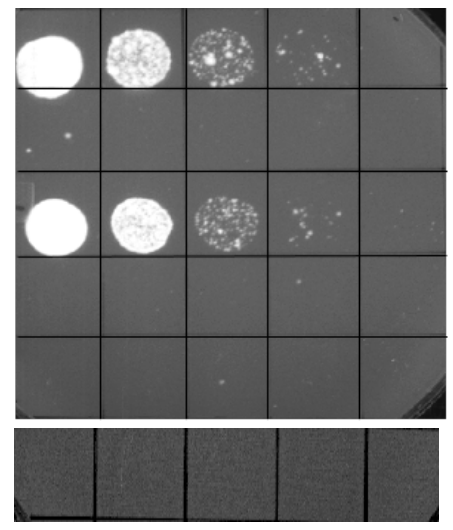

RBT $1.5 \mu \mathrm{g} / \mathrm{ml}$
C

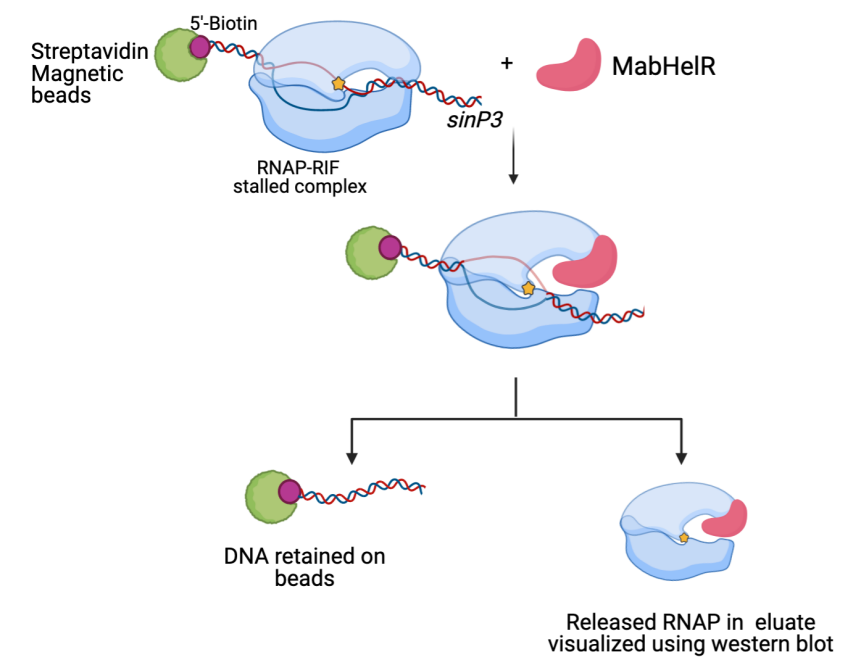

d
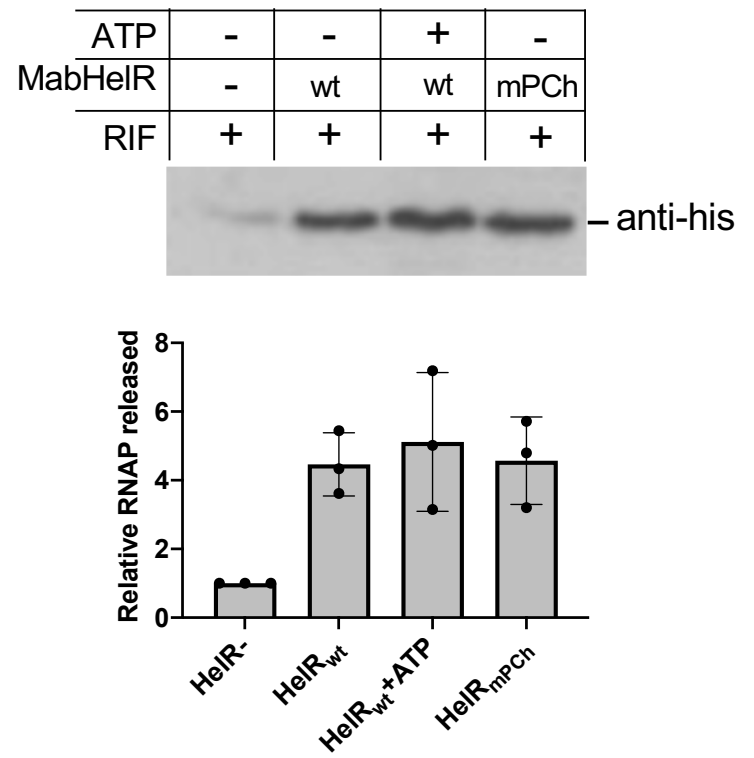

Figure 5 


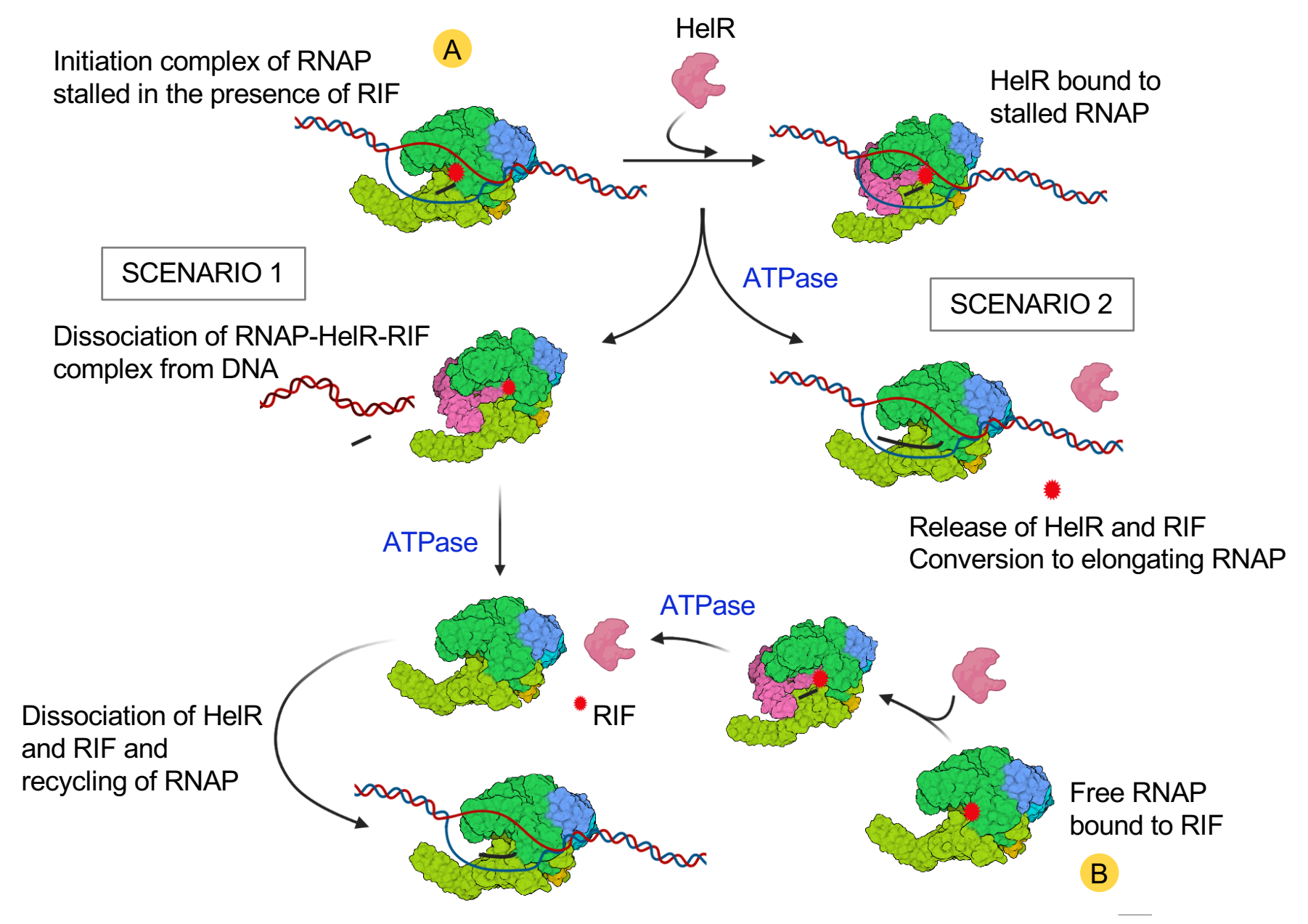

Figure 6 


\begin{tabular}{|c|c|c|}
\hline \multirow{2}{*}{ Strain } & \multicolumn{2}{|c|}{ Minimum Inhibitory Conc. ( $\mu \mathrm{g} / \mathrm{mL})$} \\
\hline & RIFAMPICIN & RIFABUTIN \\
\hline WT Mab & 64 & 2 \\
\hline$\Delta M a b \_h e / R$ & 8 & 0.5 \\
\hline$\Delta M a b \_a r r$ & 1 & 0.125 \\
\hline$\Delta M a b \_h e l R / \Delta M a b \_a r r$ & 0.125 & 0.0039 \\
\hline$\Delta M a b \_h e l R+p M H M a b-h e l R$ & $32-64$ & 2 \\
\hline
\end{tabular}

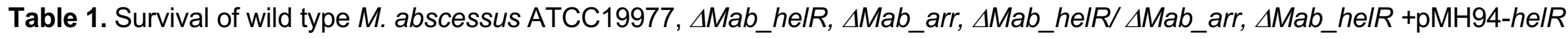
in a 2-fold dilution series of RIF and RBT in Middlebrook 7H9/OADC medium. The minimum concentration of antibiotic required to inhibit $99 \%$ of growth after 72 hours is shown. Data is representative of 3 replicates. 
a

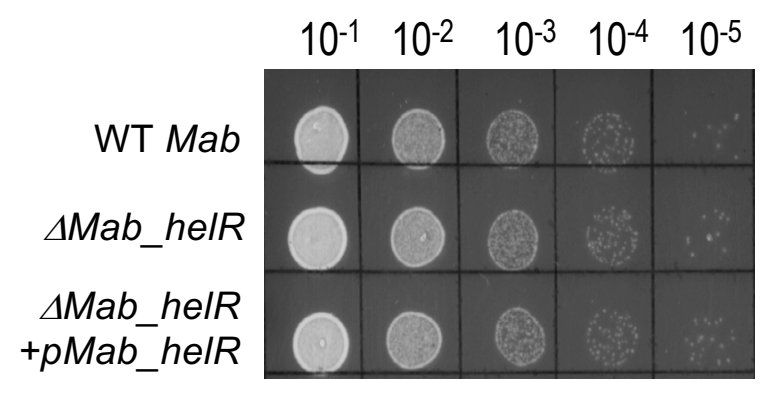

No antibiotic

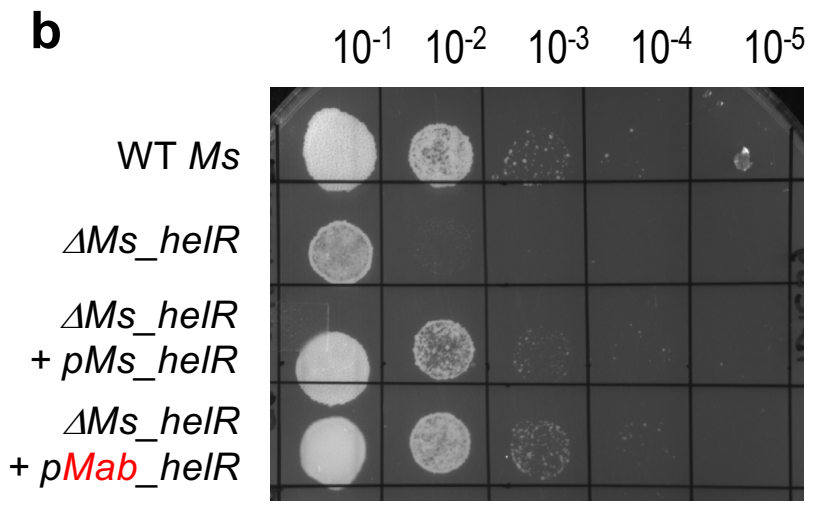

RIF $16 \mu \mathrm{g} / \mathrm{ml}$

Figure S1: a)Wild type $M$. abscessus, $\Delta$ helR and complemented strains were grown to $A_{600}$ of 0.7 and a 10 -fold dilution series was spotted on media lacking antibiotics. No growth defect was observed in the $\Delta$ helR mutant compared to WT bacteria. b) Wild type $M$. smegmatis, $\Delta M s$ s helR and $\Delta M s \_h e l R$ complemented with either HelR or Ms_helR were grown to $A_{600}$ of 0.7 and a 10 -fold dilution series was spotted on media RIF $(16 \mu \mathrm{g} / \mathrm{mL})$. Mab_helR can complement the RIF sensitivity of $\Delta M s_{-}$helR. 


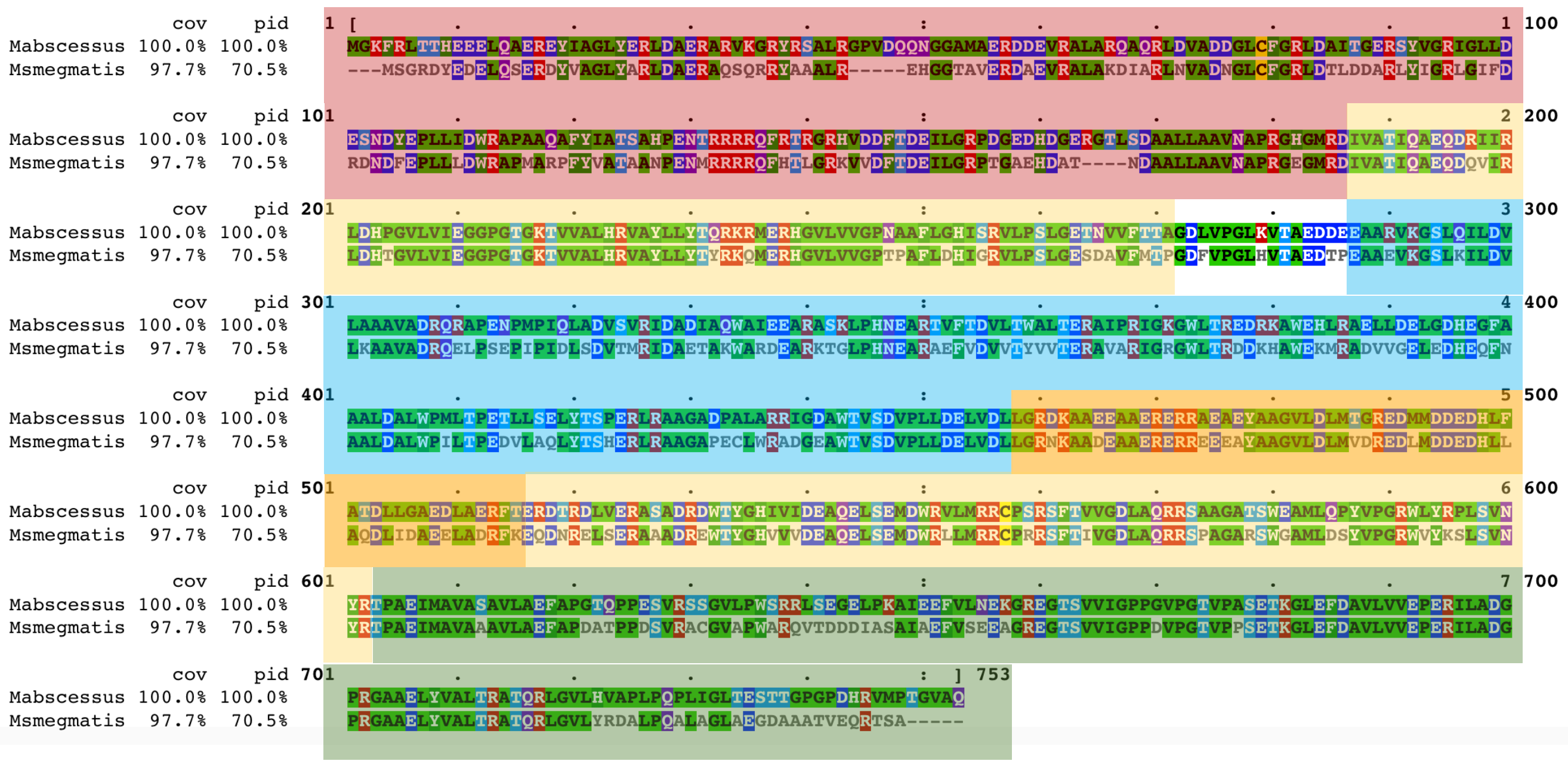

$\mathrm{N}$-terminal domain

$1 \mathrm{~A}-1$ and $1 \mathrm{~A}-2$

Clamp opening domain

PCh loop domain

Figure S2: Sequence comparison of HelR from $M$. abscessus and $M$.

smegmatis. Sequence alignment was performed using CLUSTALW. The N-terminal,

C-terminal 2A

C-terminal, $1 \mathrm{~A}-1$ and $1 \mathrm{~A}-2$ and the HelR specific domains are shaded. 
a

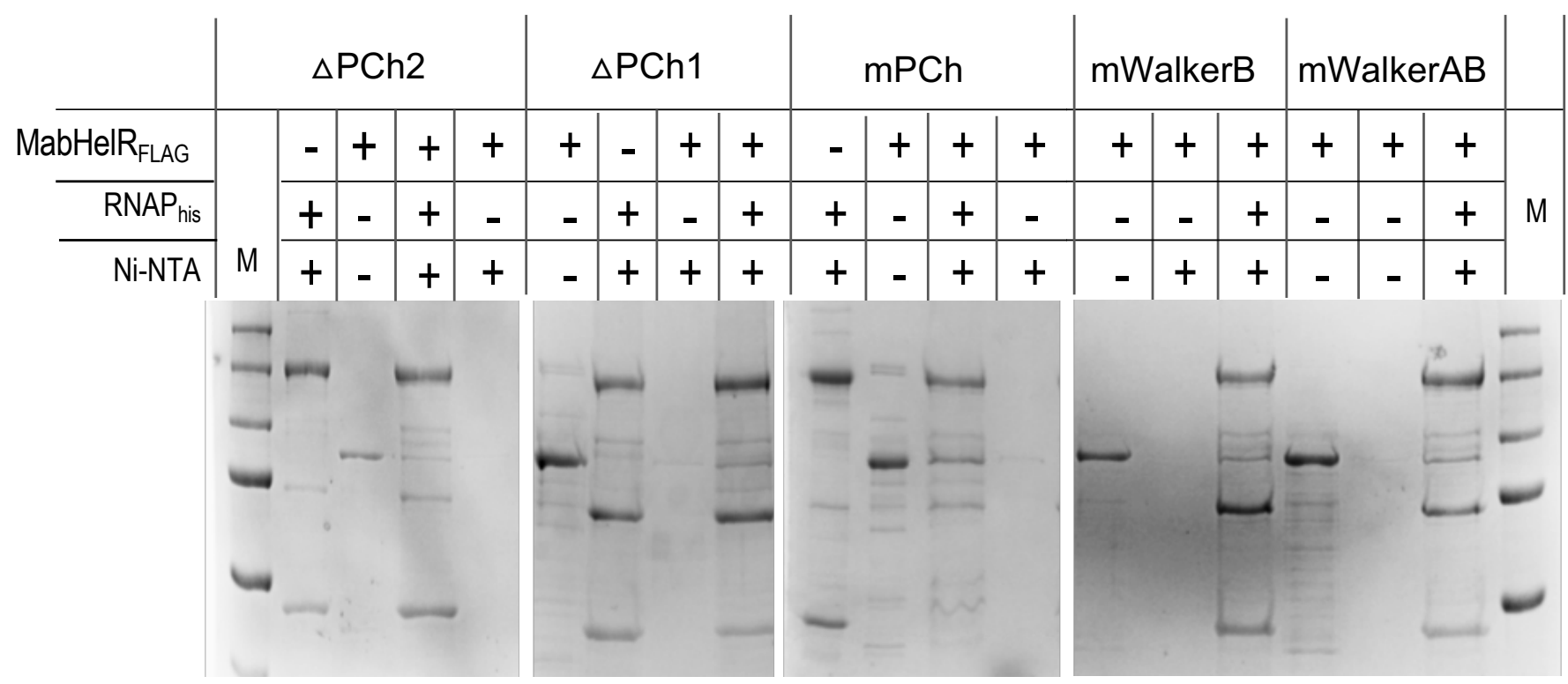

b

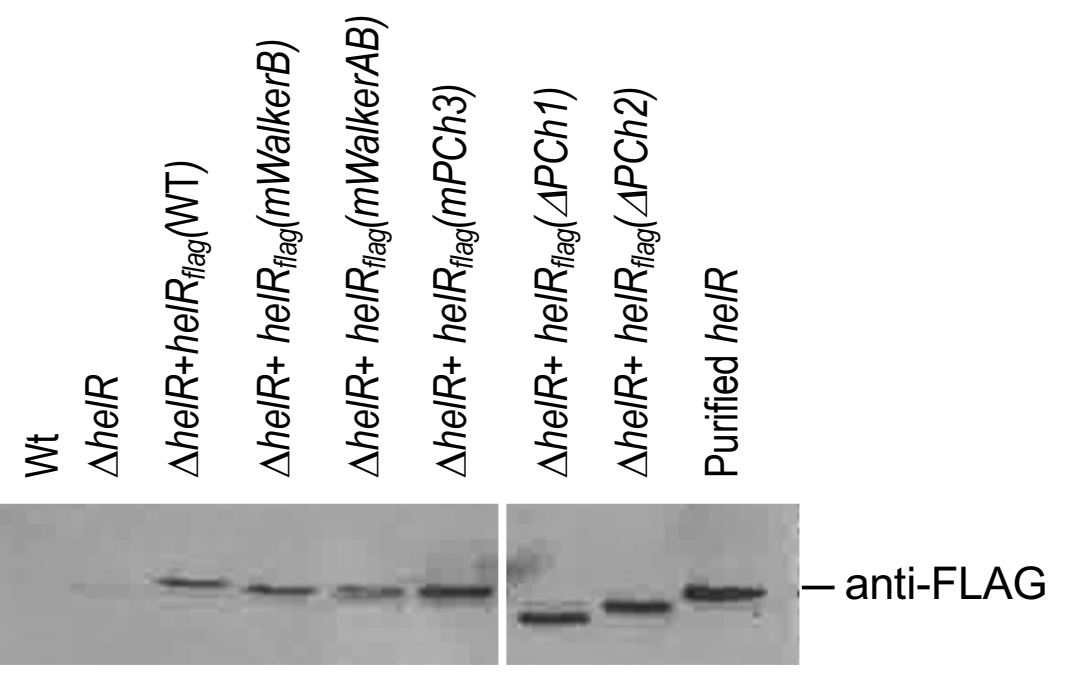

Figure S3: Expression of mutant HeIR in vivo and interaction with RNAP in vitro. b) Immunoblot with anti-FLAG antibodies of $M$. abscessus ATCC 19977, $\triangle M a b \_$helR and $\triangle M a b$ helR complemented with either WT helR $R_{F L A G}$, helR ( $\triangle$ PCh1) FLAG, helR $(\triangle \mathrm{PCh} 2)_{F L A G}$ and helR $(\mathrm{mPCh})_{\text {FLAG }}$ treated with RIF $(\overline{1} 6 \mu \mathrm{g} / \mathrm{mL})$ shows that WT and mutant proteins are induced to similar levels in the complementing strains. c) Coomassie stained gel showing elution of proteins using nickel affinity chromatography. Interaction assays were carried out using WT and mutant HeIR $\mathrm{FLAG}_{\text {with }} \mathrm{RNAP}_{\text {his }}$ as indicated and followed by binding and elution from a Ni-

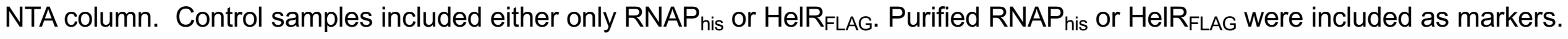


Table S1. MIC of RIF and RBT of M. abscessus clinical strains

\begin{tabular}{|c|c|c|c|c|}
\hline \multirow{2}{*}{ Strain } & Subspecies & \multirow{2}{*}{$\begin{array}{c}\text { Colony } \\
\text { Morphology }\end{array}$} & \multicolumn{2}{|l|}{ Minimum Inhibitory Conc. $(\boldsymbol{\mu g} / \mathrm{mL})$} \\
\cline { 3 - 5 } & & RIFAMPICIN & RIFABUTIN \\
\hline ATCC19977 & $\begin{array}{c}\text { M. abscessus } \\
\text { abscessus }\end{array}$ & smooth & 64 & 2 \\
\hline Mab 095 & $\begin{array}{c}\text { M. abscessus } \\
\text { massiliense }\end{array}$ & smooth & 64 & 2 \\
\hline Mab 174 & $\begin{array}{c}\text { M. abscessus } \\
\text { massiliense }\end{array}$ & smooth & 64 & 2 \\
\hline Mab 299 & $\begin{array}{c}\text { M. abscessus } \\
\text { abscessus }\end{array}$ & rough & 64 & 1 \\
\hline Mab 427 & $\begin{array}{c}\text { M. abscessus } \\
\text { abscessus }\end{array}$ & n/a & 32 & 4 \\
\hline Mab 525 & $\begin{array}{c}\text { M. abscessus } \\
\text { abscessus }\end{array}$ & rough & 128 & 2 \\
\hline Mab 549 & $\begin{array}{c}\text { M. abscessus } \\
\text { bolletii }\end{array}$ & smooth & $>64$ & 1 \\
\hline Mab 698 & $\begin{array}{c}\text { M. abscessus } \\
\text { massiliense }\end{array}$ & smooth & 64 & 2 \\
\hline Mab 998 & $\begin{array}{c}\text { M. abscessus } \\
\text { abscessus }\end{array}$ & smooth & 32 & 2 \\
\hline
\end{tabular}


Table S2. List of strains and plasmids used in the study

\begin{tabular}{|c|c|}
\hline Strain & Description \\
\hline WT M. abscessus & ATCC 19977 \\
\hline Mab:RpoA $A_{\text {his }}$ & Mab ATCC19977 with a 10X his tag at C-terminal of native $r p o A$ \\
\hline Mab:helR FLAG & Mab ATCC19977 with a 3X FLAG tag at C-terminal of native MAB_3189 \\
\hline$\Delta M a b \_h e l R$ & Unmarked deletion mutant of MAB_3189c \\
\hline$\Delta M a b \_a r r$ & Unmarked deletion mutant of MAB_0591 \\
\hline$\Delta M a b \_h e l R / \Delta M a b \_a r r$ & Unmarked Double mutant of MAB_3189c and MAB_0591 \\
\hline $\begin{array}{l}\text { MMab_helR + pMHMab- } \\
\text { helR }\end{array}$ & $\begin{array}{l}\triangle \mathrm{MAB} 3189 \text { containing a chromosomally integrated copy of MAB_3189 (WT) } \\
\text { controlled by the native promoter }\end{array}$ \\
\hline 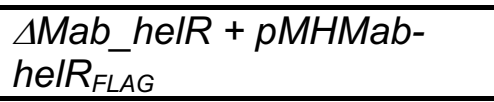 & $\begin{array}{l}\triangle \mathrm{MAB} 3189 \mathrm{c}+\text { chromosomally integrated MAB_3189 }{ }_{F L A G}(3 X \mathrm{XFAG}) \text { controlled } \\
\text { by the native promoter }\end{array}$ \\
\hline $\begin{array}{l}\triangle M a b \_h e I R+p M H M a b- \\
\text { helR (mWalkerB) } \\
\text { FLAG }\end{array}$ & $\begin{array}{l}\triangle \mathrm{MAB} \_3189 \mathrm{c}+\text { chromosomally integrated MAB_3189 }{ }_{F L A G}(\mathrm{mDEAQ}) \text { controlled } \\
\text { by the native promoter }\end{array}$ \\
\hline $\begin{array}{l}\triangle M a b \_h e l R+p M H M a b- \\
\text { helR }(m W \text { alkerAB) } \\
\text { FLAG }\end{array}$ & $\begin{array}{l}\triangle \mathrm{MAB} 3189 \mathrm{c}+\text { chromosomally integrated MAB_3189 } F L A G(\mathrm{~K} 217 \mathrm{~A} / \mathrm{mDEAQ}) \\
\text { controlled by the native promoter }\end{array}$ \\
\hline $\begin{array}{l}\triangle M a b \_h e I R+p M H M a b- \\
h e I R(\triangle P C h 1)_{F L A G}\end{array}$ & $\begin{array}{l}\triangle \mathrm{MAB} 3189 \mathrm{c}+\text { chromosomally integrated MAB_3189 } F \angle A G(\triangle 467-515) \text { controlled } \\
\text { by the native promoter }\end{array}$ \\
\hline $\begin{array}{l}\triangle M a b \_h e I R+p M H M a b- \\
\text { heIR }(\triangle P c h 2)_{F L A G}\end{array}$ & $\begin{array}{l}\triangle M A B \_3189 c+\text { chromosomally integrated MAB_3189 }{ }_{F L A G}(\triangle 486-505) \text { controlled } \\
\text { by the native promoter }\end{array}$ \\
\hline $\begin{array}{l}\triangle M a b \_h e I R+p M H M a b- \\
\text { helR }(m P c h)_{F L A G}\end{array}$ & $\begin{array}{l}\triangle \mathrm{MAB} 3189 \mathrm{c}+\text { chromosomally integrated MAB_3189 } F L A G \quad \text { (E496A/D496A ) } \\
\text { controlled by the native promoter }\end{array}$ \\
\hline Mtb+ pMH3189 & $\begin{array}{l}\text { mc }^{2} 7000+\text { containing a chromosomally integrated MAB_3189(WT) controlled by } \\
\text { the native promoter }\end{array}$ \\
\hline WT M. smegmatis & $\mathrm{mc}^{2155}$ \\
\hline$\Delta M s \_h e l R$ & Unmarked deletion mutant of MSMEG_2174 \\
\hline$\Delta M s \_h e l R+p M H M s-h e l R$ & $\begin{array}{l}\triangle \mathrm{MSMEG} 2174 \text { containing a chromosomally integrated MSMEG_2174 (WT) } \\
\text { controlled by the native promoter }\end{array}$ \\
\hline$\Delta M s \_h e l R+p M H M a b-h e l R$ & $\begin{array}{l}\triangle \mathrm{MSMEG} 2174 \text { containing a chromosomally integrated MAB_3189 (WT) } \\
\text { controlled by the native promoter }\end{array}$ \\
\hline
\end{tabular}




\begin{tabular}{|c|c|}
\hline Plasmid & Description \\
\hline pMHMab-helR & $\begin{array}{c}\text { MAB_3189 (WT) + native promoter cloned in the } \\
\text { integrating plasmid pMH94 }\end{array}$ \\
\hline pMHMab-helR $R_{F L A G}$ & MAB_3189 $(\mathrm{WT})_{\text {FLAG }}+$ native promoter cloned in pMH94 \\
\hline $\begin{array}{l}\text { pMHMab-helR } \\
(m W a l k e r B)_{F L A G}\end{array}$ & $\begin{array}{l}\text { MAB_3189(mDEAQ: D540A/E541A/Q543A })_{F L A G} \\
\text { cloned in pMH94 under control of its native promoter }\end{array}$ \\
\hline $\begin{array}{l}\text { pMHMab-helR } \\
(\text { mWalkerAB })_{F L A G}\end{array}$ & $\begin{array}{c}\text { MAB_3189 (K217A/D540A/E541A/Q543A ) FLAG Cloned } \\
\text { in pMH94 under control of its native promoter }\end{array}$ \\
\hline pMHMab-helR(APch1) $)_{\text {FLAG }}$ & $\begin{array}{c}\text { MAB_3189 }(\triangle 467-515)_{\text {FLAG }} \text { Cloned in pMH94 under } \\
\text { control of its native promoter }\end{array}$ \\
\hline pMHMab-helR $(\Delta P c h 2)_{F L A G}$ & $\begin{array}{c}\text { MAB_3189 }(\triangle 486-505) \text { cloned in pMH94 under control } \\
\text { of its native promoter }\end{array}$ \\
\hline pMHMab-helR $(m P c h)_{F L A G}$ & $\begin{array}{c}\text { MAB_3189 (E496A/D497A) })_{\text {FLAG }} \text { cloned in pMH94 } \\
\text { under control of its native promoter }\end{array}$ \\
\hline pET21a + Mab_helD-WT his & $\begin{array}{c}\text { MAB_3189 }(\mathrm{WT})_{\text {his }} \text { Cloned in pET21a under the T7 } \\
\text { promoter }\end{array}$ \\
\hline pET21a + Mab_helD-mW & $\begin{array}{c}\text { MAB_3189(D540A/E541A/Q543A })_{\text {his }} \text { cloned in pET21a } \\
\text { under the T7 promoter }\end{array}$ \\
\hline pET21a + Mab_helD- mW AB $_{\text {A }}$ & $\begin{array}{c}\text { MAB_3189 (K217A/ D540A/E541A/Q543A })_{\text {his }} \text { cloned in } \\
\text { pET21a under the T7 promoter }\end{array}$ \\
\hline pET21a + Mab_helD-WT FLAG & $\begin{array}{l}\text { MAB_3189 (WT) cloned in pET21a-FLAG under the T7 } \\
\text { promoter }\end{array}$ \\
\hline $\begin{array}{l}\text { pET21a + Mab_helD- } \\
(\Delta P \text { ch1)-FLAG }\end{array}$ & $\begin{array}{c}\text { MAB_3189 } \triangle 467-515 \text { cloned in pET21a-FLAG under } \\
\text { the T7 promoter }\end{array}$ \\
\hline $\begin{array}{l}\text { pET21a + Mab_helD- } \\
(\Delta P \text { ch2)-FLAG }\end{array}$ & $\begin{array}{c}\text { MAB_3189 } \triangle 486-505 \text { cloned in pET21a-FLAG under } \\
\text { the T7 promoter }\end{array}$ \\
\hline $\begin{array}{l}\text { pET21a + Mab_helD- } \\
\text { (mPch)-FLAG }\end{array}$ & $\begin{array}{c}\text { MAB_3189 E496A/D497A cloned in pET21a-FLAG } \\
\text { under the T7 promoter }\end{array}$ \\
\hline pETRsfDuet- Mtb $\beta \beta^{\prime}$ & pETRsfDuet containing Mtb rpoB and rpoC \\
\hline pETRsfDuet- Mtb $\alpha \omega$ & pETRsfDuet containing Mtb rpoA-his 10 and rpoZ \\
\hline pET21a+Mtb $\sigma^{A}$ & pET21a containing Mtb SigA \\
\hline $\mathrm{pET} 21 \mathrm{a}+\mathrm{Mab} \sigma^{\mathrm{A}}$ & pET21a containing Mab SigA \\
\hline
\end{tabular}

\section{SinP3 DNA:}

gaagaaatacgaaaatatttacttttaaacaaaaagtctgctcatcctggtccggcagccagaagtcataccgtaaatcctttctgaatgtgctataatatcacAaggaaggtgatgac attgattggccagcgtattaaacaataccgtaaagaaaaaggctactcactatcagaactag

(start site is in upper case and underlined) 